\title{
Genome wide identification and functional characterization of two LC-PUFA biosynthesis elongase (elovl8) genes in rabbitfish (Siganus canaliculatus)
}

Yang $\mathrm{Li}^{\mathrm{a} \dagger}$, Zhengyong Wen ${ }^{\mathrm{c}},{ }^{\mathrm{d} \dagger}$, Cuihong You ${ }^{\mathrm{a}}$, Zhiyong Xie ${ }^{\mathrm{a}}$, Douglas R. Tocher ${ }^{\mathrm{e}}$, Yueling Zhanga ${ }^{\mathrm{a}}$, Shuqi Wang ${ }^{\mathrm{a}}$, Yuanyou $\mathrm{Li}^{\mathrm{b}^{*}}$

${ }^{a}$ Guangdong Provincial Key Laboratory of Marine Biotechnology, Institute of Marine Sciences, Shantou University, Shantou 515063, China

${ }^{b}$ College of Marine Sciences of South China Agricultural University \& Guangdong Laboratory of Lingnan Modern Agriculture, Guangzhou, 510642, China

${ }^{c}$ BGI Education Center, University of Chinese Academy of Sciences, Shenzhen 518083, China

${ }^{d}$ Shenzhen Key Lab of Marine Genomics, Guangdong Provincial Key Lab of Molecular Breeding in Marine Economic Animals, BGI Academy of Marine Sciences, BGI Marine, BGI, Shenzhen 518083, China

${ }^{e}$ Institute of Aquaculture, Faculty of Natural Sciences, University of Stirling, Stirling FK9 4LA, Scotland, UK

\section{*Correspondence Author:}

Prof. Yuanyou Li, Ph.D. (E-mail: yyli16@scau.edu.cn; Tel: 020-87571321)

Shuqi Wang, Ph.D. (E-mail: sqw@ stu.edu.cn; Tel: 0754-86500614)

${ }^{\dagger}$ These authors contributed equally to this work. 


\section{Abstract}

Elongases of very long-chain fatty acids (Elovls) catalyze the rate-limiting step of the elongation pathway that results in net 2-carbon elongation of pre-existing fatty acyl chains. As a set of crucial enzymes involved in the long-chain polyunsaturated fatty acids (LC-PUFA) biosynthesis, Elovls of fish have been investigated extensively in recent years. In the present study, we first identified two novel fish-specific elovl genes (named as elovl8a and elovl8b) from the herbivorous marine teleost rabbitfish (Siganus canaliculatus) by genomic survey and molecular cloning methods. Subsequently, their functional characteristics, tissue distribution patterns and transcriptional changes in response to different nutritional states were investigated. Full- length coding sequences of the elovl $8 a$ and elovl $8 b$ genes were 804 and 792 bp, encoding 267 and 263 amino acids, respectively. Multiple alignment, genomic synteny and phylogenetic analyses further suggested that elovl8 genes were unique to teleosts. Functional characterization by heterologous expression in yeast showed that Elovl8b could elongate C18 (18:2n-6, 18:3n-3 and 18:4n-3) and C20 (20:4n-6 and 20:5n-3) polyunsaturated fatty acids (PUFA) to longer-chain polyunsaturated fatty acids (LC-PUFA) whereas Elovl8a lacked this ability. In vitro, the expression of elovl8b but not elovl8a in rabbitfish hepatocytes was significantly up-regulated by incubation with 18:2n-6, 18:3n-3, 20:4n-6 and 20:5n-3, respectively. In vivo, compared with fish oil, dietary vegetable oil enriched in C18 PUFA enhanced the expression of elovl $8 b$ in rabbitfish brain, liver, intestine and gill. These findings suggest that elovl8b but not elovl8a is a novel active member of the Elovl protein family involved in the LC-PUFA biosynthesis pathway in rabbitfish, and provide novel insight into the mechanisms of LC-PUFA biosynthesis in teleost.

Keywords: elovl8; Functional characterization; LC-PUFA biosynthesis; Rabbitfish (Siganus canaliculatus) 


\section{Introduction}

Long-chain ( $\mathrm{n}>\mathrm{C} 20$ ) polyunsaturated fatty acids (LC-PUFA), especially the omega-3 (n-3) LC-PUFA eicosapentaenoic (EPA; 20:5n-3) and docosahexaenoic (DHA; 22:6n-3) acids, are major components of complex lipid molecules and are also involved in numerous critical biological processes in vertebrates, including maintenance of cellular membrane structure, energy metabolism, gene regulation and cellular signaling, and can promote cardiovascular health and immune function (Tocher, 2010; Vagner and Santigosa, 2011). Fish, especially marine fish, are major sources of n-3 LC-PUFA in human diets (Kromhout et al., 2012). With the rapid decline of wild fishery stocks, aquaculture now plays a more important role than wild fisheries in providing fish and seafood for human consumption and it is expected to supply the majority of seafood to satisfy future increased demand (Clavelle et al., 2019). Traditionally, farmed fish were fed with diets containing high levels of marine fishmeal (FM) and fish oil (FO) to ensure good growth rates of the fish and high levels of n-3 LC-PUFA in the flesh. Nowadays, vegetable oils (VO) are widely used in aquafeeds, which reduces the level of n-3 LC-PUFA in farmed fish and, consequently, decreases their nutritional value (Sprague et al., 2016; Henriques et al., 2014). This has prompted interest in elucidating the mechanisms underlying the endogenous LC-PUFA biosynthetic pathways in teleost fish, particularly farmed marine species that have been investigated extensively in recent years (Castro et al., 2016; Monroig and Kabeya, 2018).

In vertebrates, LC-PUFA can be biosynthesized from C18 PUFA precursors, linoleic acid (LA, 18:2n-6) and $\alpha$-linolenic acid (ALA, 18:3n-3), through the concerted action of fatty acyl desaturases (Fads) and Elovl proteins (Castro et al., 2016; Xue et al., 2014). Elovls are key microsomal enzymes involved in the biosynthesis of LC-PUFA from C18 PUFA. Elovls catalyze the condensation reaction, which is the rate- limiting step in the two-carbon elongation of pre-existing fatty acyl chains (Nugteren, 1965). In mammals, seven members of the elovl 
family were identified based on the presence of specific motifs in their protein sequences (Jakobsson et al., 2006; Guillou et al., 2010). Specifically, elovl1, elovl3, elovl6 and elovl7 have the ability to elongate saturated and monounsaturated fatty acids (MUFA), while elovl2, elovl4 and elovl5 are involved in elongating PUFA (Jakobsson et al., 2006; Monroig et al., 2010; Guillou et al., 2010).

Investigation of the Elovl enzymes involved in LC-PUFA biosynthesis, focused on Elovl2, Elovl4 and Elovl5, enabled a better understanding of the PUFA elongation pathways in teleosts. The zebrafish (Danio rerio) elovl5 was the first cloned elovl gene that was functionally characterized as a critical enzyme in the elongation step of LC-PUFA biosynthesis from a fish species (Agaba et al., 2004). Subsequently, elovl5 was cloned and characterized in numerous fish species (Agaba et al., 2005; Hastings et al., 2004; Zheng et al., 2009; MohdYusof et al., 2010; Morais et al., 2009, 2011; Kim et al., 2012; Monroig et al., 2012, 2013; Gregory et al., 2010, 2014). These studies confirmed that elovl5 in fish had the ability to preferentially elongate C18 (18:4n-3 and 18:3n-6) and C20 (EPA and arachidonic acid, ARA, 20:4n-6) PUFA, with only low activity towards C22 PUFA (22:5n-3 and 22:4n-6), which was similar to mammalian and invertebrate homologues (Leonard et al., 2000, 2002; Li et al., 2016). However, elovl 2 was only isolated from a few fish species, and shown to mainly elongate C20 and C22 PUFA with only low ability to elongate C18 PUFA (Morais et al., 2009; Monroig et al., 2009; Gregory and James, 2014).

Elovl4 is the most recent member of the Elovl family to be studied in teleost fish, which includes two isoforms of elovl4a and elovl $4 b$. The elovl $4 a$ was first identified and functionally characterized in zebrafish, which indicated it had the ability to efficiently elongate saturated fatty acids up to C36 (Monroig et al., 2010). However, elovl4b was shown to have the ability to efficiently elongate acyl chain- lengths of saturated fatty acids and C20 LC-PUFA (ARA and EPA) up to C36 saturated and very long-chain PUFA (VLC-PUFA) in various fish species, 
respectively (Carmona-Antoñanzas et al., 2011; Jin et al., 2017; Kabeya et al., 2015; Li et al., 2017a, 2017b; Monroig et al., 2010, 2012, 2011; Yan et al., 2018).

In addition to elovl $4 a$ and elovl $4 b$, two further elovl4-like genes (termed as elovl $4 c-1$ and elovl4c-2) were cloned from Atlantic cod (Gadus morhua), and phylogenetic analysis showed they clustered separately from elovl $4 a$ or elovl $4 b$ genes (Xue et al., 2014). Indeed, surveys of fish genomes revealed that there might be similar elovl genes that have been annotated as "elovl4" (or "elovl4- like") in many fish species such as Atlantic salmon (Salmo salar, XP_014071374), channel catfish (Ictalurus punctatus, XP_017324302), Nile tilapia (Oreochromis niloticus, XP_005479178.1), and rabbitfish. Interestingly, two similar isoforms had been found in the zebrafish genome (NP_001191453 and NP_001070061) and they were annotated as elovl8a and elovl $8 b$, respectively. Although the relevant annotation and the phylogenetic analysis have identified these novel genes as elovls, their functions are unknown. Therefore, it is important to investigate whether these genes are involved in LC-PUFA biosynthesis.

Rabbitfish, an economically important herbivorous marine teleost fish species widespread along the Indo-West Pacific coast, was the first marine teleost demonstrated to have capability for LC-PUFA biosynthesis from C18 PUFA (Li et al., 2010). Genes encoding key enzymes with all the activities required for LC-PUFA biosynthesis including $\Delta 4$ fads 2 , bifunctional $\Delta 6 / \Delta 5 \mathrm{fads} 2$, elovl4 and elovl5, have been cloned and functionally characterized in this species, which makes rabbitfish a good model for studying the mechanisms of LC-PUFA biosynthesis (Li et al., 2010; Monroig et al., 2012). In order to expand our knowledge of LCPUFA biosynthesis in rabbitfish, we performed a series of bioinformatic analyses on this fish species, and identified a novel member of the elovl gene family, consisting of two isoforms, elovl8a and elovl8b. The cDNAs were functionally characterized, and tissue distribution patterns and transcriptional changes in various tissues and rabbitfish hepatocytes (SCHL) under 
different nutritional states were determined. Our findings suggest that rabbitfish Elovl8b can elongate LC-PUFA, while Elovl8a lacks this ability. To our knowledge, this is the first comprehensive report of teleost elovl8 genes. The results can provide a better understanding of the mechanisms of LC-PUFA biosynthesis in vertebrates and may contribute to the optimization and/or enhancement of LC-PUFA biosynthesis in fishes.

\section{Materials and methods}

\subsection{Experimental animals and sample collection}

Fish were treated in accordance with the National Institutes of Health guide for the care and use of Laboratory animals (NIH Publications No. 8023, revised 1978) and approved by the Institutional Animal Care and Use Committee of Shantou University (Guangdong, China).

The feeding trial was carried out at Nan Ao Marine Biology Station (NAMBS) of Shantou University, Southern China. Three hundred rabbitfish juveniles (average initial body weight around $15 \mathrm{~g}$, sex visually indistinguishable) were captured from the coast near NAMBS. After one month of acclimation in an indoor seawater (32 ppt) tank at NAMBS, fish with initial average body weight of $25.07 \pm 1.43 \mathrm{~g}$ were then randomly distributed into 6 tanks with 20 fish per tank in triplicate per treatment. Throughout the trial, the fish were fed two experimental diets with approximatively $32 \%$ crude protein and $8 \%$ crude lipid with the latter supplied by either fish oil (FO) or vegetable oil (VO). The details of the formulation and proximate composition of the experimental diets were shown in Supplemental Table 1. At the end of the 8-week feeding trial, six fish from each tank were randomly selected and brain, liver, intestine and gills were sampled for comparative gene expression analysis. Additionally, during the acclimation period, six fish were randomly selected for molecular cloning and tissue distribution studies. The dissected tissues were immediately frozen in liquid nitrogen and stored at $-80{ }^{\circ} \mathrm{C}$ until further use.

\subsection{RNA isolation and $q P C R$}


Total RNA was extracted with Trizol reagent (Invitrogen, Carlsbad, CA, USA) according to manufacturer's protocol. The concentration and quality of total RNA were confirmed by spectrophotometer (NanoDrop 2000, Thermo Scientific, USA) and $1 \mu$ g total RNA was reverse transcribed to cDNA using the QuantiTect® Reverse Transcription kit (Takara Biotech, Dalian, China). Determination of mRNA expression levels was performed by quantitative real-time PCR (qPCR) in a LightCycler® 480 thermocycler (Roche, Germany) in a total volume of 20 $\mu$ with the LightCycler® 480 SYBR Green I Master (Roche, Germany) following the manufacturer's protocol. All amplification reactions were carried out in triplicate and a nontemplate control was also included in each run. The relative expression level of mRNA was normalized with that $18 \mathrm{~S}$ rRNA, and calculated using the comparative threshold cycle method (Livak and Schmittgen, 2012; Wen et al., 2019). The primer pairs used for RT-PCR are given in Supplemental Table 2.

\subsection{Molecular cloning of elovl8 cDNAs from rabbitfish}

Based on genomic DNA sequences identified from our genome database and cDNA sequences from our transcriptome database (unpublished), we designed four pairs of primers (Supplemental Table 2) to amplify the full- length cDNA sequences of rabbitfish elovl8a and elovl $8 b$ genes by using liver tissue as template. PCR amplifications were carried out on an Applied Biosystems Veriti Thermal Cycler using $2 \times$ Phanta Max Master Mix (Vazyme Biotech, Nanjing, China) for high fidelity amplification. The target products were purified using TIAN quick mini purification kit (Tiangen Biotech, Beijing, China), cloned into pMD ${ }^{\mathrm{TM}}$ 18-T vector (TaKaRa Biotech, Dalian, China) and subsequently sequenced (Sangon Biotech, Shanghai, China).

2.4 Bioinformatic analysis, data processing and phylogenetic analysis

The obtained sequences were blasted on the NCBI database for annotation, and the valid rabbitfish elovl8a and elovl8b cDNA sequences were submitted to NCBI (MN807637 
and MN807638) and used for further bioinformatic analyses. The open reading frames (ORFs) of the two genes were predicted using ORF finder (https://www.ncbi.nlm.nih.gov/orffinder/), and the putative protein sequences were translated using Primer Premier 5.0 software. Functional motifs were identified according to previous literature. Subsequently, multiple protein sequences alignment was performed using BioEdit software (Hall et al., 2011). Additionally, synteny and gene structures of fish elovl8 genes were compared on the basis of a comparative genomic survey to validate the existent of the fish elovl8 genes.

To explore the phylogenetic position of the fish Elovl8s, we constructed a phylogenetic tree based on a dataset of protein sequences. All Elovl protein sequences were downloaded from NCBI or Ensembl database except for the rabbitfish Elovl8s, and then multiple alignment was performed using CLUSTAL X2.1 (Larkin et al., 2007). After alignment, Mega 6.0 software (Tamura et al., 2013) was used to construct the phylogenetic tree using the neighbour-joining (NJ) method, and JTT + G was selected as the best model according to the model calculation. Meanwhile, a nonparametric bootstrap analysis with 1,000 resampling replicates was used to assess the robustness of the tree topology. Mimachlamys nobilis Elovl- like protein was selected as the outgroup.

\subsection{Functional characterization in yeast}

Liver cDNA was used to amplify the PCR fragments corresponding to the open reading frame (ORF) of the rabbitfish elovl $a$ and elovl $8 b$, using $2 \times$ Phanta Max Master Mix (Vazyme Biotech, Nanjing, China) according to manufacturer's protocol. Special primer pairs with restriction sites (underlined) for ORF cloning are listed in Supplemental Table 2. PCR conditions consisted of an initial denaturing step at $95^{\circ} \mathrm{C}$ for $3 \mathrm{~min}$, followed by 35 cycles of denaturation at $95^{\circ} \mathrm{C}$ for $10 \mathrm{~s}$, annealing at $62^{\circ} \mathrm{C}\left(\right.$ elovl8a) or $65^{\circ} \mathrm{C}($ elovl $8 b)$ for 10 s, extension at $72{ }^{\circ} \mathrm{C}$ for 30 s, followed by a final extension at $72{ }^{\circ} \mathrm{C}$ for $8 \mathrm{~min}$. DNA fragments were purified (TIAN quick mini purification kit, Tiangen Biotech), digested with the corresponding 
restriction endonucleases (New England Biolabs, Inc., USA) and ligated to a similarly restricted pYES2 yeast expression vector (Invitrogen, UK). The purified recombinant plasmids containing the putative elovl8a or elovl8b ORFs were used to transform Saccharomyces cerevisiae competent cells (S.c EasyComp Transformation Kit, Invitrogen).

Transformation and selection of yeast with recombinant plasmids (pYES2-elovl8a or pYES2-elovl8b) and yeast culture were performed according to the methods described by Monroig et al. (2012). Recombinant yeasts were incubated in media containing one of the following fatty acid substrates: 18:3n-3, 18:2n-6, 18:4n-3, 18:3n-6, 20:5n-3, 20:4n-6, 22:5n-3 and 22:4n-6 (Cayman, Ann Arbor, USA). The final concentrations of fatty acid substrates added to the yeast cultures were as following: $0.5(\mathrm{C} 18), 0.75(\mathrm{C} 20)$ and $1.0(\mathrm{C} 22) \mathrm{mM}$. As control treatment, yeast transformed with empty pYES2 was cultured under the same conditions. After 2 days, yeast was harvested and washed for further analyses.

\subsection{Fatty acid analysis by GC-MS}

Total lipid of yeast samples was extracted according to Yan et al. (2018) and fatty acid methyl esters (FAME) were prepared and purified according to method described by Christie (2003). FAME were identified and quantified by gas chromatography coupled with a mass spectrometer (GC-MS) (2010- ultra, Shimadzu, Japan) as described previously (Hastings et al., 2001; Agaba et al., 2004). Conversions of PUFA substrates were calculated by the proportion of substrate fatty acid (FA) converted to elongated FA products, as [individual product area/ (all products areas + substrate area) $] \times 100$.

\subsection{Cell culture and fatty acid incubation}

The rabbitfish S. canaliculatus hepatocyte line (SCHL) was successfully established previously (Liu et al., 2017). Before the experiment, SCHL cells were cultured at $28{ }^{\circ} \mathrm{C}$ in Dulbecco's modified Eagle's medium/nutrient F12 (DMEM/F12, Gibco, Life Technologies, USA) containing $20 \mathrm{mM}$ 4-(2-hydroxyethyl) piperazine-1-ethanesulphonic acid (HEPES, 
Sigma-Aldrich, USA), $10 \%$ fetal bovine serum (FBS, Gibco), $0.5 \%$ rainbow trout (Oncorhynchus mykiss) serum (Caisson Labs), penicillin (100 U ml-1, Sigma-Aldrich), and streptomycin (100 U ml-1, Sigma-Aldrich).

Fatty acid/ BSA complexes of PUFA including LA, ALA, ARA, EPA and DHA (Cayman, Ann Arbor, USA) at $10 \mathrm{mM}$ concentration were prepared according to Ou et al. (2001) and stored at $-20^{\circ} \mathrm{C}$. The SCHL cells were seeded into six-well plates at a density of $1.0 \times 106$ cells per well in DMEM/F12 supplemented with $5 \% \mathrm{FBS}$ and $0.1 \%$ rainbow trout serum. After $24 \mathrm{~h}$, cells were incubated for $2 \mathrm{~h}$ in serum- free DMEM/F12 and then exposed to fresh DMEM/F12 medium containing LA, ALA, ARA, EPA or DHA at $100 \mu \mathrm{M}$ in triplicate per treatment. In addition, $0.1 \%$ BSA was used as control for PUFA treatments. After incubation for $24 \mathrm{~h}$, the cells were lysed with Trizol reagent (Invitrogen) for total RNA isolation.

\subsection{Statistical Analysis}

All the data are presented as means \pm SEM. The qPCR expression data were analyzed by one-way analysis of variance (ANOVA) followed by Tukey's multiple comparison test or Student's t test using Origin 7.0. Statistical significances were considered to be significant if $P$ $<0.05$.

\section{Results}

\subsection{Molecular identification of two rabbitfish elovl8 genes}

The rabbitfish elovl8a cDNA contains an 804-bp ORF encoding 267 putative amino acid residues (Fig. 1A), while the rabbitfish elovl $8 b$ cDNA contains a 792-bp ORF encoding 263 putative amino acid residues (Fig. 1B). Both deduced protein sequences possess six transmembrane $\alpha$-helix domains, which are considered to be highly conserved among vertebrates including fish. Moreover, four conserved motifs characteristic of elongases were 
identified in the two cDNAs (blue type in Fig 1A and B), respectively. In addition, ER retrieval signals were also identified at the C-terminals in both cDNAs.

\subsection{Multiple alignments of the Elovl8s among fishes}

Aligning of multiple amino acid sequences could be helpful for better understanding of structural and functional properties of the examined proteins. Here, multiple alignments of Elovl8a and Elovl8b among several fishes were performed. We observed that Elovl8s are highly conserved across fishes, which share similar structural characteristics (such as six conserved transmembrane $\alpha$-helix domains, four conserved motifs of elongases, and three highly conserved cysteine residues; see Fig. 2). It is notable that the peptide sequences from the position 1 to 21 are variable and non-conservative (Fig. 2), and Elovl8a is approximately 4 residues longer than its paralog Elovl8b at the N-terminal (Fig. 2).

\subsection{Synteny and gene structure comparisons of the elovl8 genes in vertebrates}

In order to verify the existence of both elovl8 genes in teleosts, comparative genomic synteny analysis among vertebrates was performed (Fig. 3). Neither elovl8a nor elovl8b were found in amphibians, reptiles, birds or mammals. Two elovl8 genes were discovered in zebrafish and rabbitfish (Fig. 3A and B), and they share similar gene orders respectively. Furthermore, only a single copy of elovl8a was determined in spotted gar and Asian arowana (Fig. 3A), and elovl $8 b$ was only determined in Mexican tetra and channel catfish (Fig. 3B). The elovl8a and elovl8b genes were differentially localized with the elovl8a gene surrounded by seppb and zswim5 (Fig. 3A), while the elovl8b was commonly located between mutyh and glis 1 (Fig. 3B). Interestingly, an elovl4-like gene was found in the similar genomic locus as elovl8a in an ancient fish, the coelacanth (Fig. 3A).

Comparative gene structure analysis was performed to discover the difference between elovl8a and elovl8b genes. Results showed that the elovl8a gene consisted of eight exons and seven introns in spotted gar, zebrafish and rabbitfish, while the Asian arowana elovl8a gene 
possessed seven exons and six introns (Fig. 4A). The exon length of elovl8a was conserved in the five middle exons but was variable in the other exons. In contrast, the gene structures of elovl $8 b$ in all the fish species were highly conserved, consisting of eight exons and seven introns, with identical ORF length (Fig. 4B).

\subsection{Phylogenetic analysis}

To better understand evolutionary relationships among the teleost elovl8s, phylogenetic analysis using the Neighbor Joining (NJ) method was performed. The phylogenetic tree showed five clusters for elovl8, elovl4, elovl2, elovl6 and elovl5, with the elovl8 cluster showing closest relationship to the elovl4 cluster (Fig.5). Meanwhile, the elovl8 cluster was further subdivided into two clades of elovl8a and elovl8b subtypes (Fig. 5). The rabbitfish elovl8a and elovl8b were grouped into the elovl $8 a$ and elovl $8 b$ clades, and shared close relationships with Amazon molly elovl 8 a and fugu elovl8b, respectively.

\subsection{Tissue distribution patterns of rabbitfish elovl8s}

Tissue distribution patterns of the two elovl8 genes in rabbitfish were determined by qPCR. Twelve tissues including stomach, liver, spleen, heart, gonad, brain, kidney, intestine, muscle, adipose, eye and gill were analyzed. The rabbitfish elovl 8 a was widely transcribed in all examined tissues, and expression was highest in heart and spleen, with the rank order being: heart $>$ spleen $>$ adipose $>$ stomach $>$ muscle $>$ intestine $>$ kidney $>$ gill $>$ eye $>$ liver $>$ gonad $>$ brain (Fig. 6A). Similarly, the rabbitfish elovl8b was also extensively distributed in all tissues, with highest expression in brain, eye, and liver with the rank order being: brain > eye $>$ liver $>$ gonad $>$ adipose $>$ gill $>$ spleen $>$ intestine $>$ kidney $>$ heart $>$ stomach $>$ muscle $($ Fig . $6 B)$.

\subsection{Functional characterizations of rabbitfish Elovl8a and Elovl8b}

Potential functions of the two putative elovl8 elongases in rabbitfish were determined by heterologous expression in yeast $S$. cerevisiae grown in medium supplemented one of the 
following fatty acid substrates: 18:2n-6, 18:3n-3, 18:4n-3, 18:3n-6, 20:4n-6, 20:5n-3, 22:5n-3 and 22:4n-6. The fatty acid composition of yeast transformed with the empty vector (pYES2) showed that the recombinant yeast lacked PUFA elongase activity (data not shown). In yeast transformed with the rabbitfish Elovl8a, no additional peaks were detected with any of the added substrate PUFA and so it lacked the ability to convert any of these PUFA into longer chain PUFA (Table 1). In contrast, GC-MS analyses revealed that rabbitfish Elovl8b had the ability to elongate C18 PUFA to C20 PUFA, with conversion rates for 18:2n-6 to 20:2n-6, $18: 3 n-3$ to $20: 3 n-3$ and $18: 4 n-3$ to $20: 4 n-3$ of $2.0 \%, 3.7 \%$ and $3.7 \%$, respectively (Table 1 ). Similarly, rabbitfish Elovl8b was able to elongate C20 PUFA to C22 PUFA, with conversion rates for $20: 4 n-6$ and $20: 5 n-3$ to $22: 4 n-6$ and $22: 5 n-3$ of $2.0 \%$ and $3.2 \%$, respectively.

3.7 Effect of different fatty acids on the expressions of elovl8a and elovl8b in SCHL cells

The relative expression levels of elovl8a and elovl8b genes in SCHL cells incubated with different fatty acids, including LA, ALA, ARA, EPA and DHA, were determined using qPCR. Results showed that the transcription level of elovl8a was not significanltly altered by different fatty acid substrates (Fig. 7A). In contrast, the mRNA expression of elovl8b was significantly increased in SCHL cells incubated with LA, ALA, ARA and EPA, and slightly reduced in SCHL cells incubated with DHA compared with the control group of SCHL cells incubated with BSA alone.

\subsection{Effect of dietary lipid sources on the elovl8b expression in rabbitfish}

To investigate transcriptional changes of the rabbitfish elovl $8 b$ gene in response to different dietary lipid sources, we measured the elovl $8 b$ mRNA levels in the brain, liver, intestine and gill of rabbitfish. The results showed that the expression of elovl $8 b$ in rabbitfish brain (Fig. 8A), liver (Fig. 8B), intestine (Fig. 8C) and gill (Fig. 8D) of the fish fed with VO was significantly higher than those fed with FO.

\section{Discussion}


In present study, two novel elovl genes were found in rabbitfish and identified as elovl8a and elovl8b). The complete coding sequence of rabbitfish elovl8a was 804-bp encoding a putative 267 -aa protein and the coding sequence of rabbitfish elovl8b was 792-bp encoding a putative 263-aa protein. The newly cloned elovl8s of rabbitfish showed relatively low sequence identity with rabbitfish elovl4 (49\% $51 \%)$ and elovl5 (41.9\% $44.9 \%)$, and higher identity with zebrafish elovl8s $(70.38 \%$ 81.4\%) (Supplemental Table 3). Multiple alignment suggested that the rabbitfish elovl8s were similar to its paralogs of the elovl $4 \mathrm{~b}$ and elovl5 (Monroig et al., 2012), and possess the typical features of the elongase family including the predicted transmembrane domains, the histidine box $(\mathrm{HXXHH})$, and the canonical $\mathrm{C}$ terminal ER retrieval signal (Jakobsson et al., 2006; Monroig et al., 2010; Xue et al., 2014). These findings suggested that rabbitfish elovl8s might represent a new member of Elovl protein family, which we hypothesized could be involved in LC-PUFA biosynthesis in teleosts.

In order to verify our hypothesis, we analyzed the genomic synteny and gene structures of rabbitfish elovl8s using a comparative genomic survey method, and then compared these data with elovl8s of several different fish species. We found that elovl8s were present in some teleosts but not in amphibians, reptiles, birds, and mammals. In addition, an elovl4- like gene was found at a similar genetic locus with fish elovl8a in an ancient fish, the coelacanth. This suggested that elovl8 genes may be unique to teleosts, and that they might have arisen from a common ancestral gene, which is the elovl4- like gene of sarcopterygii. Two paralogs of elovl8 were identified in some teleost species, which might be a result of the teleost-specific whole genome duplication event that is well known in fish evolution and is considered as an important driving force of biological evolution (Glasauer and Neuhauss, 2014; Jaillon et al., 2004; Meyer and Van de Peer, 2005, Castro et al., 2016). Furthermore, the rabbitfish elovl8a was clearly different from the elovl $8 b$, with localization at different genetic loci and gene structure, which confirmed the existence of two elovl8 genes in the rabbitfish. Interestingly, the older lineages 
of teleosts (spotted gar and Asian arowana) have retained only a single copy, specifically an elovl8a with features that are more in common with the sarcopterygii, whereas the more recently evolved teleosts (Mexican tetra and channel catfish) also have only one copy, but these were elovl $8 b$ with more derived features. These results suggested that the elovl8a may appear earlier than $e l o v l 8 b$ in fish evolution, and they may have differential physiological functions. The different gene structures of elovl8a and elovl8b gene may suggest or reflect differential physiological roles. Compared with the elovl 8 a gene, the gene structures of elovl $8 b$ were more conserved, consisting of the same number of exon introns, and same ORF length. These findings suggest that the physiological functions of elovl8 might have been differentiated in the evolutionary process, and that the function of elovl $8 b$ in LC-PUFA biosynthesis was more conserved than any potential activity of elovl8a.

Phylogenetic analysis showed that the elovl8s clade was obviously different from other elongases, which separated from the subgroup of elovl4, elovl2, elovl6 and elovl5 clades, suggested elovl8s might be a novel type of the Elovl protein family ubiquitously existent in teleost. Meanwhile, the elovl8s cluster was further subdivided into two clades of elovl8a and elovl8b, indicated two different elovl8 isoforms are widely spread in fishes. Furthermore, we observed that the elovl8 subgroup shared a closer relationship with elovl4 cluster which was located at the root of tree, suggested the elovl8s might derive from elovl4 in fish. This may explain why elovl8 had been previously annotated as an elovl4-like gene (Xue et al., 2014). A comprehensive evolutionary study on elovls performed in chordates recently showed that the diversification of elovls (elovl2, elovl5 and elovl4) involved in LC-PUFA biosynthesis in vertebrates was expanded from elovl 2/5 and elovl4 in chordate ancestry (Castro et al., 2016). However, searches in silico strongly suggest that the elovl2 gene may have lost (e.g. silenced) in the vast majority of marine fish species (Morais et al., 2009; Monroig et al., 2011). In contrast, most of fishes appear to possess at least one copy of elovl8s, and two isoforms of 
elovl8 are presented in rabbitfish. It is uncertain what mechanism leads fishes to retain elovl8 instead of elovl2 gene selectively, but it is reasonable to speculate that the ubiquitous existence elovl8 genes might act in the biosynthesis of LC-PUFA in teleost in order to compensate the physiological functions of lost elovl2.

Tissue distribution experiment indicated that elovl8s were widely distributed in all examined tissues in rabbitfish, and the elovl8a was highly expressed in heart and spleen while elovl $8 b$ was mainly distributed in the brain, eye (possibly retina) and liver. The different distribution patterns suggested these two elovl8 isoforms could play various roles in rabbitfish. The high transcription of rabbitfish elovl8b but not elovl8a in the brain, eye and liver further supported the hypothesis that elovl8b may play an important role in LC-PUFA biosynthesis, as these tissues are major metabolic sites for the biosynthesis of LC-PUFA in teleosts (CarmonaAntoñanzas et al., 2011; Monroig et al., 2010, 2011, 2012). Interestingly, the tissue distribution of elovl $8 b$ in rabbitfish was similar to the tissue distribution of elovl5 in the majority of fish species including rabbitfish (Gregory et al., 2010; Mohd-Yusof et al., 2010; Zheng et al., 2009; Monroig et al., 2012), which may suggest that elovl8b could play a similar role to elovl5 in other fish species.

Functional characterization in yeast showed that rabbitfish Elovl8a had no ability to utilize all the added substrates including C18-PUFAs, C20-PUFAs and C22-PUFAs, suggesting Elovl8a do not participate in the process of LC-PUFA biosynthesis in rabbitfish. Meanwhile, these findings further supported the evolutionary status and distribution pattern of the elovl8a in rabbitfish. However, the elovl8a was extensively transcribed in majority of examined tissues, suggesting the protein may play important roles in other aspects and its exact roles are still unknown and more researches are needed to declare. Additionally, Elovl8b was shown to have the ability to convert C18 PUFA (18:2n-6, 18:3n-3 and 18:4n-3) and C20 PUFA (20:4n-6 and 20:5n-3) to longer-chain PUFAs, suggesting Elovl8b should be involved in LC- 
PUFA biosynthesis in rabbitfish. Usually, elovl5 and elovl4 have been regarded as the primary enzymes for the elongation of C18 and C20 PUFAs in fish (Monroig et al., 2012; Castro et al., 2016). In our previous study, we found rabbitfish Elovl4 and Elovl5 possessed all elongation properties in LC-PUFA biosynthesis with high conversion efficiency (Monroig et al., 2012). The present study reveals that except for elovl5 and elovl4, the elovl $8 b$ also participates in LCPUFA biosynthesis. However, the low efficiency of Elovl8b to utilize the fatty acid substrates, suggesting Elovl8b may play as an alternative enzyme involved in LC-PUFA biosynthesis in the main metabolism tissues. Moreover, elovl8b was found to be widely expressed, suggesting this gene may also play important roles in other physiological processes.

To further clarify the functional differences between rabbitfish elovl8a and elovl $8 \mathrm{~b}$ in LC-PUFA biosynthesis, expression levels of elovl8a and elovl8b were determined in SCHL cells incubated with different PUFA, including LA, ALA, ARA, EPA and DHA. We found the transcriptional level of elovl8a was not significantly altered by different fatty acid substrates, which further confirmed that the rabbitfish elovl8a lacked the ability to biosynthesize LCPUFA. In contrast, the mRNA expression of elovl $8 b$ was significantly up-regulated by LA, ALA, ARA and EPA, and slightly down-regulated by DHA. These data indicated that elovl $8 b$ could be actively involved in the LC-PUFA biosynthesis pathway as it's expression was stimulated by the C18, LA and ALA, and C20, ARA and EPA, pathway substrate fatty acids and inhibited by the pathway product fatty acid, DHA.

Additionally, in vivo experiment showed that the expression levels of elovl $8 \mathrm{~b}$ in rabbitfish tissues were significantly affected by dietary lipid source, similar to other elovl genes in other fish species (Kuah et al., 2015; Xue et al., 2014; Xie et al., 2016). We herein showed that the expression of elovl $8 \mathrm{~b}$ in rabbitfish brain, liver, intestine and gill in rabbitfish fed VO were significantly higher than those fish fed FO. Compared with the FO that is rich in EPA and DHA, VO have no n-3 LC-PUFA, but are generally rich in C18 PUFA, LA and/or ALA 
(Izquierdo et al., 2003; Raso and Anderson, 2003; Tocher, 2003). Therefore, the LC-PUFA biosynthesis pathway of rabbitfish was stimulated by dietary VO, which might be caused by a compensatory mechanism to meet the lower dietary levels of essential LC-PUFAs. Rabbitfish elovl $8 b$, as a potential elongase gene involved in LC-PUFA biosynthesis, was higher expressed in fish fed with VO, which might be a physiological adaptation of this fish to the deficiencies of dietary LC-PUFA through increased LC-PUFA biosynthesis. These findings were consistent with our previous report that the transcriptions of LC-PUFAs biosynthesis related genes ( $\Delta$ $4 \mathrm{fad}, \Delta \quad 6 / 5 \mathrm{fad}$ and elovl5) were significantly higher in rabbitfishes fed with VO than those of that in fishes fed with FO (Xie et al., 2015), suggesting elovl8b is also involved in LCPUFAs biosynthesis.

\section{Conclusion}

In the present study, we identified elovl $8 a$ and elovl $8 b$ genes from the rabbitfish and functionally investigated their potential roles in response to different nutritional states for the first time. Our data confirmed that the elovl8 genes are unique to teleosts, and their physiological functions have been differentiated. The rabbitfish Elovl8b retained the ability to elongate C18 (18:2n-6, 18:3n-3 and 18:4n-3) and C20 (20:4n-6 and 20:5n-3) PUFA to longerchain fatty acids whereas Elovl8a has lost this ability. Based on these results, we can conclude that the elovl $8 b$ as a novel member of Elovl protein family involved in the LC-PUFA biosynthesis pathway in rabbitfish. Therefore, this systematic study of elovl8's elongation function towards C18 and C20 PUFAs, provided an alternative pathway for LC-PUFA biosynthesis in fish species (Fig. 9). Furthermore, the functional characterization of Elovl8b protein increases the number of Elovl enzymes already known to participate in LC-PUFA synthesis in fish, which provides a novel insight into the mechanisms of LC-PUFA biosynthesis in teleost. 


\section{Acknowledgments}

This work was financially supported by the National Key R\&D Program of China (2018YFD0900400), National Natural Science Foundation of China (No. 31873040). Natural Science Foundation of Guangdong Province (2018A030313910), China Agriculture Research System (CARS-47), and Guangdong Agriculture Research System (2019KJ150).

\section{References}

Agaba, M., Tocher, D.R., Dickson, C.A., Dick, J.R., Teale, A.J., 2004. Zebrafish cDNA encoding multifunctional fatty acid elongase involved in production of eicosapentaenoic (20:5n-3) and docosahexaenoic (22:6n-3) acids. Mar. Biotechnol. 6(3), 251-261.

Agaba, M.K., Tocher, D.R., Zheng, X., Dickson, C.A., Dick, J.R., Teale, A.J., 2005. Cloning and functional characterisation of polyunsaturated fatty acid elongases of marine and freshwater teleost fish. Comp. Biochem. Physiol. B Biochem. Mol. Biol. 142(3), 342352.

Carmona-Antoñanzas, G., Monroig, Ó., Dick, J.R., Davie, A., Tocher, D., 2011. Biosynthesis of very long-chain fatty acids (C>24) in Atlantic salmon: Cloning, functional characterisation, and tissue distribution of an Elovl4 elongase. Comp. Biochem. Physiol. B Biochem. Mol. Biol. 159(2), 122-129.

Castro, L.F.C., Tocher, D.R., Monroig, O., 2016. Long-chain polyunsaturated fatty acid biosynthesis in chordates: Insights into the evolution of Fads and Elovl gene repertoire. Prog. Lipid Res. 62, 25-40.

Christie, W.W., 2003. Lipid Analysis, 3rd ed., Oily Press, Bridgwater.

Clavelle, T., Lester, S.E., Gentry, R., Froehlich, H.E., 2019. Interactions and management for the future of marine aquaculture and capture fisheries. Fish Fish. 20(2), 368-388.

Glasauer, S.M., Neuhauss, S.C., 2014. Whole-genome duplication in teleost fishes and its evolutionary consequences. Mol. Genet. Genomic. 289(6), 1045-1060.

Gregory, M.K., James, M.J., 2014. Rainbow trout (Oncorhynchus mykiss) Elov15 and Elovl2 differ in selectivity for elongation of omega-3 docosapentaenoic acid. BBA-Mol. Cell Biol. L. 1841(12), 1656-1660. 
Gregory, M.K., See, V.H., Gibson, R.A., Schuller, K.A., 2010. Cloning and functional characterisation of a fatty acyl elongase from southern bluefin tuna (Thunnus maccoyii). Comp. Biochem. Physiol. B Biochem. Mol. Biol. 155(2), 178-185.

Guillou, H., Zadravec, D., Martin, P.G., Jacobsson, A., 2010. The key roles of elongases and desaturases in mammalian fatty acid metabolism: Insights from transgenic mice. Prog. Lipid Res. 49(2), 186-199.

Hall, T., Biosciences, I., Carlsbad, C., 2011. BioEdit: an important software for molecular biology. GERF Bull Biosci. 2(1), 60-61.

Hastings, N., Agaba, M., Tocher, D.R., Leaver, M.J., Dick, J.R., Sargent, J.R., Teale, A., 2001. A vertebrate fatty acid desaturase with $\Delta 5$ and $\Delta 6$ activities. P. Nat. Acad. Sci. USA. 98(25), 14304-14309.

Hastings, N., Agaba, M.K., Tocher, D.R., Zheng, X., Dickson, C.A., Dick, J.R., Teale, A.J., 2004. Molecular cloning and functional characterization of fatty acyl desaturase and elongase cDNAs involved in the production of eicosapentaenoic and docosahexaenoic acids from $\alpha$-linolenic acid in Atlantic salmon (Salmo salar). Mar. Biotechnol. 6(5), 463-474.

Henriques, J., Dick, J.R., Tocher, D.R., Bell, J.G., 2014. Nutritional quality of salmon products available from major retailers in the UK: content and composition of n-3 long-chain PUFA. Brit. J. Nutr. 112(6), 964-975.

Izquierdo, M.S., Obach, A., Arantzamendi, L., Montero, D., Robaina, L., Rosenlund, G., 2003. Dietary lipid sources for seabream and seabass: growth performance, tissue composition and flesh quality. Aquacult. Nutr. 9(6), 397-407.

Jaillon, O., Aury, J.M., Brunet, F., Petit, J.L., Stange-Thomann, N., Mauceli, E., Bouneau, L., Fischer, C., Ozouf-Costaz, C., Bernot, A., Nicaud, S., Jaffe, D., Fisher, S., Lutfalla, G., Dossat, C., Segurens, B., Dasilva, C., Salanoubat, M., Levy, M., Boudet, N., Castellano, S., Anthouard, V., Jubin, C., Castelli, V., Katinka, M., Vacherie, B., Biemont, C., Skalli, Z., Cattolico, L., Poulain, J., De Berardinis, V., Cruaud, C., Duprat, S., Brottier, P., Coutanceau, J.P., Gouzy, J., Parra, G., Lardier, G., Chapple, C., McKernan, K.J., McEwan, P., Bosak, S., Kellis, M., Volff, J.N., Guigo, R., Zody, M.C., Mesirov, J., Lindblad-Toh, K., Birren, B., Nusbaum, C., Kahn, D., RobinsonRechavi, M., Laudet, V., Schachter, V., Quetier, F., Saurin, W., Scarpelli, C., Wincker, P., Lander, E.S., Weissenbach, J., Roest Crollius, H., 2004. Genome duplication in the teleost fish Tetraodon nigroviridis reveals the early vertebrate proto-karyotype. Nature. 431, 946-957. 
Jakobsson, A., Westerberg, R., Jacobsson, A., 2006. Fatty acid elongases in mammals: their regulation and roles in metabolism. Prog. Lipid Res. 45(3), 237-249.

Jin, M., Monroig, O., Navarro, J.C., Tocher, D.R., Zhou, Q.C., 2017. Molecular and functional characterisation of two elovl4 elongases involved in the biosynthesis of very long-chain (> C24) polyunsaturated fatty acids in black seabream Acanthopagrus schlegelii. Comp. Biochem. Physiol. B Biochem. Mol. Biol. 212, 41-50.

Kabeya, N., Yamamoto, Y., Cummins, S. F., Elizur, A., Yazawa, R., Takeuchi, Y., Haga, Y., Satoh, S., Yoshizaki, G., 2015. Polyunsaturated fatty acid metabolism in a marine teleost, Nibe croaker Nibea mitsukurii: Functional characterization of Fads2 desaturase and Elov15 and Elov14 elongases. Comp. Biochem. Physiol. B Biochem. Mol. Biol. 188, 37-45.

Kim, S.H., Kim, J.B., Jang, Y.S., Kim, S.Y., Roh, .H., Kim, H.U., Lee, K.R., Park, J.S., 2012. Isolation and functional characterization of polyunsaturated fatty acid elongase (AsELOVL5) gene from black seabream (Acanthopagrus schlegelii). Biotechnol. Lett. 34(2), 261-268.

Kromhout, D., Yasuda, S., Geleijnse, J.M., Shimokawa, H., 2012. Fish oil and omega-3 fatty acids in cardiovascular disease: do they really work? Eur. Heart J. 33(4), 436-443.

Kuah, M.K., Jaya-Ram, A., Shu-Chien, A.C., 2015. The capacity for long-chain polyunsaturated fatty acid synthesis in a carnivorous vertebrate: Functional characterisation and nutritional regulation of a Fads2 fatty acyl desaturase with $\Delta 4$ activity and an Elovl5 elongase in striped snakehead (Channa striata). BBA-Mol. Cell Biol. L. 1851(3), 248-260.

Larkin, M.A., Blackshields, G., Brown, N.P., Chenna, R., McGettigan, P.A., McWilliam, H., Valentin, F., Wallace, I.M., Wilm, A., Lopez, R., Thompson, J.D., Gibson, T.J., Higgins, D.G., 2007. Clustal W and Clustal X version 2.0. Bioinformatics. 23, 29472948.

Leonard, A.E., Bobik, E.G., Dorado, J., Kroeger, P.E., Chuang, L.T., Thurmond, J. M., Parker-Barnes, J.M., Das, T., Huang, Y.S., Mukerji, P., 2000. Cloning of a human cDNA encoding a novel enzyme involved in the elongation of long-chain polyunsaturated fatty acids. Biochem. J. 350(3), 765-770.

Leonard, A.E., Kelder, B., Bobik, E.G., Chuang, L.T., Lewis, C.J., Kopchick, J.J., Mukerji, P., Huang, Y.S., 2002., Identification and expression of mammalian long-chain PUFA elongation enzymes. Lipids 37(8), 733-740. 
Li, S., Monroig, Ó., Navarro, J.C., Yuan, Y., Xu, W., Mai, K., Tocher, D.R., Ai, Q., 2017. Molecular cloning and functional characterization of a putative Elovl4 gene and its expression in response to dietary fatty acid profiles in orange-spotted grouper Epinephelus coioides. Aquac. Res. 48(2), 537-552.

Li, S., Monroig, O., Wang, T., Yuan, Y., Carlos Navarro, J., Hontoria, F., Liao, K., Tocher, D.R., Mai, K., Xu, W., Ai, Q., 2017. Functional characterization and differential nutritional regulation of putative Elovl5 and Elovl4 elongases in large yellow croaker (Larimichthys crocea). Sci Rep. 7, 2303.

Li, W., Feng, Z., Song, X., Zhu, W., Hu, Y., 2016. Cloning, expression and functional characterization of the polyunsaturated fatty acid elongase (ELOVL5) gene from sea cucumber (Apostichopus japonicus). Gene 593(1), 217-224.

Li, Y., Monroig, O., Zhang, L., Wang, S., Zheng, X., Dick, J. R., You, C.H., Tocher, D. R., 2010. Vertebrate fatty acyl desaturase with $\Delta 4$ activity. P. Nat. Acad. Sci. USA. 107(39), 16840-16845.

Liu, Y., Zhang, Q.H., Dong, Y.W., You, C.H., Wang, S.Q., Li, Y.Q., Li, Y.Y., 2017.

Establishment of a hepatocyte line for studying biosynthesis of long-chain polyunsaturated fatty acids from a marine teleost, the white-spotted spinefoot Siganus canaliculatus. J. Fish Boil. 91(2), 603-616.

Livak, K., Schmittgen, T., 2000. Analysis of relative gene expression data using real-time quantitative per and the 2- $\triangle \Delta \mathrm{ct}$ method. Methods 25(4).

Meyer, A., Van de Peer, Y., 2005. From 2R to 3R: evidence for a fish-specific genome duplication (FSGD). Bioessays 27(9), 937-945.

Mohd-Yusof, N.Y., Monroig, O., Mohd-Adnan, A., Wan, K.L., Tocher, D.R., 2010. Investigation of highly unsaturated fatty acid metabolism in the Asian sea bass, Lates calcarifer. Fish Physiol. Bioche. 36(4), 827-843.

Monroig, Ó., Kabeya, N., 2018. Desaturases and elongases involved in polyunsaturated fatty acid biosynthesis in aquatic invertebrates: a comprehensive review. Fisheries Sci. 84(6), 911-928.

Monroig, Ó., Rotllant, J., Cerdá-Reverter, J.M., Dick, J.R., Figueras, A., Tocher, D.R., 2010. Expression and role of Elovl4 elongases in biosynthesis of very long-chain fatty acids during zebrafish Danio rerio early embryonic development. BBA-Mol. Cell Biol. L. 1801(10), 1145-1154. 
Monroig, Ó., Rotllant, J., Sánchez, E., Cerdá-Reverter, J.M., Tocher, D.R., 2009. Expression of long-chain polyunsaturated fatty acid (LC-PUFA) biosynthesis genes during zebrafish Danio rerio early embryogenesis. BBA-Mol. Cell Biol. L. 1791(11), 10931101.

Monroig, Ó., Tocher, D.R., Hontoria, F., Navarro, J.C., 2013. Functional characterisation of a Fads2 fatty acyl desaturase with $\Delta 6 / \Delta 8$ activity and an Elov15 with C16, C18 and C20 elongase activity in the anadromous teleost meagre (Argyrosomus regius). Aquaculture 412, 14-22.

Monroig, Ó., Wang, S., Zhang, L., You, C., Tocher, D.R., Li, Y., 2012. Elongation of longchain fatty acids in rabbitfish Siganus canaliculatus: Cloning, functional characterisation and tissue distribution of Elovl5-and Elovl4- like elongases. Aquaculture 350, 63-70.

Monroig, Ó., Webb, K., Ibarra-Castro, L., Holt, G.J., Tocher, D.R., 2011. Biosynthesis of long-chain polyunsaturated fatty acids in marine fish: Characterization of an Elov14like elongase from cobia Rachycentron canadum and activation of the pathway during early life stages. Aquaculture 312(1-4), 145-153.

Morais, S., Monroig, O., Zheng, X., Leaver, M.J., Tocher, D.R.. 2009. Highly unsaturated fatty acid synthesis in Atlantic salmon: characterization of ELOVL5-and ELOVL2like elongases. Mar. Biotechnol. 11(5), 627-639.

Morais, S., Mourente, G., Ortega, A., Tocher, J.A., Tocher, D.R., 2011. Expression of fatty acyl desaturase and elongase genes, and evolution of DHA: EPA ratio during development of unfed larvae of Atlantic bluefin tuna (Thunnus thynnus L.). Aquaculture 313(1-4), 129-139.

Nugteren, D.H., 1965. The enzymic chain elongation of fatty acids by rat- liver microsomes. BBA-Lipid Lipid Met. 106(2), 280-290.

Ou, J., Tu, H., Shan, B., Luk, A., DeBose-Boyd, R.A., Bashmakov, Y., Goldstein, J.L., Brown, M.S., 2001. Unsaturated fatty acids inhibit transcription of the sterol regulatory element-binding protein-1c (SREBP-1c) gene by antagonizing liganddependent activation of the LXR. P. Nat. Acad. Sci. USA. 98(11), 6027-6032.

Raso, S., Anderson, T.A., 2003. Effects of dietary fish oil replacement on growth and carcass proximate composition of juvenile barramundi (Lates calcarifer). Aquac. Res. 34(10), 813-819.

Sprague, M., Dick, J.R., Tocher, D.R., 2016. Impact of sustainable feeds on omega-3 longchain fatty acid levels in farmed Atlantic salmon, 2006-2015. Sci. Rep. 6, 21892. 
Tamura, K., Stecher, G., Peterson, D., Filipski, A., Kumar, S., 2013. MEGA6: molecular evolutionary genetics analysis version 6.0. Mol. Boil. Evol. 30(12), 2725-2729.

Tocher, D.R., 2010. Fatty acid requirements in ontogeny of marine and freshwater fish. Aquac. Res. 41(5), 717-732.

Tocher, D.R., 2003. Metabolism and functions of lipids and fatty acids in teleost fish. Rev. Fish. Sci. 11(2), 107-184.

Vagner, M., Santigosa, E., 2011. Characterization and modulation of gene expression and enzymatic activity of delta-6 desaturase in teleosts: A review. Aquaculture 315(1-2), 131-143.

Wen, Z. Y., Wang, J., Bian, C., Zhang, X., Li, J., Peng, Y., Zhan, Q., Shi, Q., Li, Y. Y., 2019. Molecular cloning of two kcnk3 genes from the Northern snakehead (Channa argus) for quantification of their transcriptions in response to fasting and refeeding. Gen. Comp. Endocrinol. 281, 49-57.

Xie, D., Wang, S., You, C., Chen, F., Tocher, D.R., Li, Y., 2015. Characteristics of LCPUFA biosynthesis in marine herbivorous teleost Siganus canaliculatus under different ambient salinities. Aquacult. Nutr. 21, 541-551.

Xie, D., Chen, F., Lin, S., You, C., Wang, S., Zhang, Q., Monroig, Ó., Tocher, D.R., Li, Y., 2016. Long-chain polyunsaturated fatty acid biosynthesis in the euryhaline herbivorous teleost Scatophagus argus: Functional characterization, tissue expression and nutritional regulation of two fatty acyl elongases. Comp. Biochem. Physiol. B Biochem. Mol. Biol. 198, 37-45.

Xue, X., Feng, C. Y., Hixson, S.M., Johnstone, K., Anderson, D.M., Parrish, C.C., Rise, M.L., 2014. Characterization of the fatty acyl elongase (elovl) gene family, and hepatic elovl and delta-6 fatty acyl desaturase transcript expression and fatty acid responses to diets containing camelina oil in Atlantic cod (Gadus morhua). Comp. Biochem. Physiol. B Biochem. Mol. Biol. 175, 9-22.

Yan, J., Liang, X., Cui, Y., Cao, X., Gao, J., 2018. Elovl4 can effectively elongate C18 polyunsaturated fatty acids in loach Misgurnus anguillicaudatus. Biochem. Bioph. Res. Co. 495(4), 2637-2642.

Zheng, X., Ding, Z., Xu, Y., Monroig, O., Morais, S., Tocher, D.R., 2009. Physiological roles of fatty acyl desaturases and elongases in marine fish: characterisation of cDNAs of fatty acyl $\Delta 6$ desaturase and elovl5 elongase of cobia (Rachycentron canadum). Aquaculture 290(1-2), 122-131. 



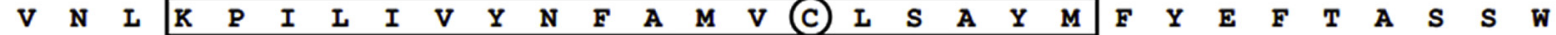

TTGGCCAGATACAGCGTGCTGTGCCAGCCAGTGGACTACAGTAGCAGCCCACTAGCCATGAGGATGGCAAGAGTGTGCTGGTGGTTCTAT

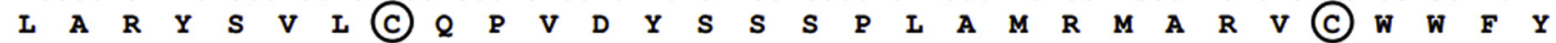
TTCTCCAAAGTGATAGAGCTCAGTGACACTATATTTTTCATCCTGAGGAAAAAGAACAATCAGCTGACTTTCCTCCACGTCTACCACCAC

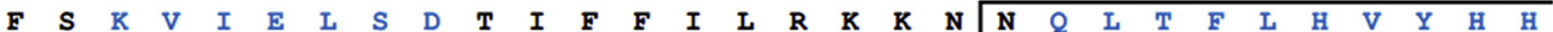
GCCACCATGATCTTCAACTGGTGGGCTGGGGTCAAATATGTGGCTGGTGGCCAATCATTCCTAATCGGTTTGATCAACTCCCTGGTCCAC

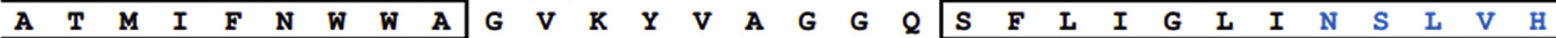
GTAGTCATGTACTTGTACTACGGCCTGGCAGCTTTTGGACCAAGCATGACTAAATACCTCTGGTGGAAACGTTACCTCACATGCCTGCAG

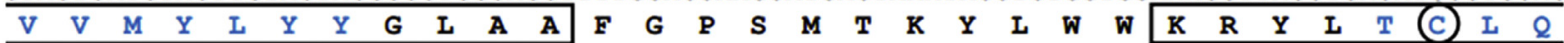
CTGCTGCAGTTTTTCATTGTGACCATCCACACCATTTACAACCTGTTTGCCGACTGTGACTTTCCTGACTCTATGAACGCGGTGGTGTTG

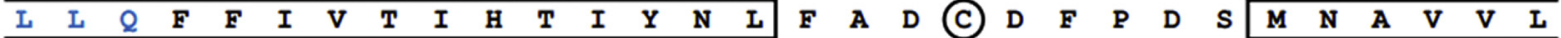
GCCTACTCTCTCAGCCTCATCGCACTCTTCAGTAACTTCTACTACCAGAGCTACCTCGCCAAGAAGAAGAGCAAGAAGACCTAG

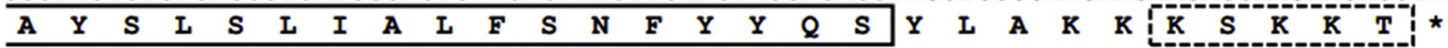

ATGGCTTCTGCATGGGAAGATGTCCTGTCAGTGTACCAGAGCTATCTGGATAACGGAGACAAGAGGACGGATCCATGGCTGCTGGTCTAC

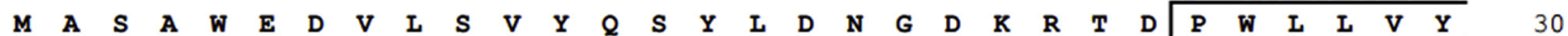
TCCCCAGTCCCCGTGGCACTCATCTTCCTGGTCTACCTCTTTGTGGTCTGGCTCGGCCCTCGTCTGATGCACCACAAACAACCTGTTGAC 180

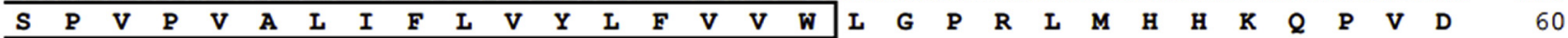
CTCAAAGCTGTCCTCATAGTTTATAATTTCGCCATGGTTGGCCTGTCTGCGTACATGTGCTATGAGTTCCTGGTCACTTCCTGGCTTTCG 270

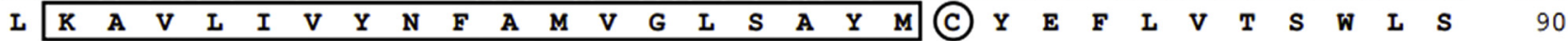
AACTACAGCTTCCTTTGCCAGCCTGTAGATTACAGCAGCAGTCCGCTGGCGATGAGGATGGCCAGAGCTTGCTGGTGGTTCTTCTTCTCT 360

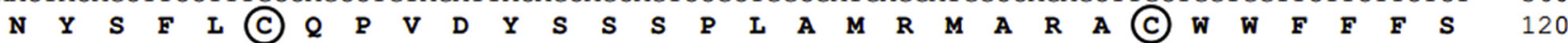
AAGATCATCGAACTCAGCGACACGATCTTCTTCATCCTGAGGAAGAAGAACAGTCAGGTGACTTTCCTTCACGTTTACCACCACGCCACC 450

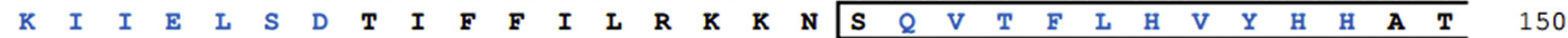
ATGATTTTCAACTGGTGGGCGGGAATCAAGTATGTGGCCGGCGGACAGTCGTTCTTCATCGGCGTGGTCAACTCCTTCGTCCACGTTGTG 540

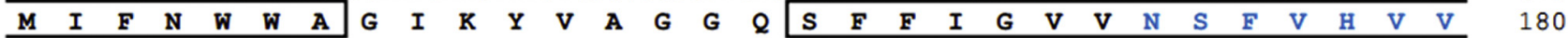
ATGTACTCGTACTACGGCCTGGCCGCCTTGGGCCCTCACATGCAGAAGTACCTGTGGTGGAAGAGGTACATCACCTCTCTGCAGCTGGTG 630

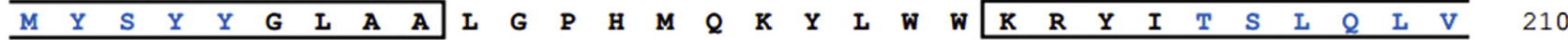
CAGTTCGTGCTCTTCCTCGTGCACACGGGTTACAACCTGTTCGCTGAGTGTGACTTCCCAGACTCCATGAACTTGTTTGTGTTCAGTTAC 720

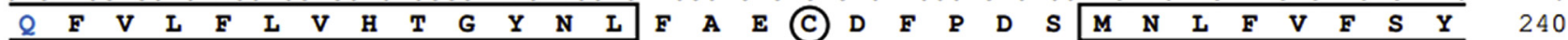
TGTGTCACCCTCATCATCCTCTTCAGCAACTTCTACTACCAAAGCTACGTCAACAAGAAGAAACAGAAATAA

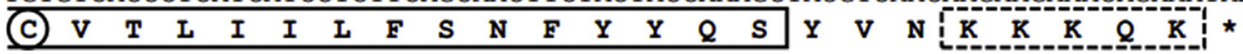

Fig. 1. Complete coding sequences and deduced protein sequences of the elovl8a (A) and elovl8b (B) genes in S.canaliculatus. Positions of nucleotides and amino acids are labeled on both sides. Transmembrane conserved domains are boxed. Amino acids sequences in blue type represent the conserved motifs of elongases. Cysteines in the two proteins are signed by circles. The putative endoplasmic reticulum (ER) retrieval signal at the C-terminus is marked by dotted boxes. Asterisk (*) represents the stop codon. 
Poecilia Formosa ELOVL8a Siganus canaliculatus ELOVL8a Danio rerio ELOVL8a Lepisosteus oculatus ELOVL8a Danio rerio ELOVL8b Astyanax mexicanus ELOVL8b Ictalurus punctatus ELOVL8b Poecilia formosa ELOVL8b Siganus canaliculatus ELOVL8b Clustal Consensus

Poecilia formosa ELOVL8a Siganus canaliculatus ELOVL8a Danio rerio ELOVL8a Lepisosteus oculatus ELOVL8a Danio rerio ELOVL8b Astyanax mexicanus ELOVL8b Ictalurus punctatus ELOVL8b Poecilia formosa ELOVL8b Siganus canaliculatus ELOVL8b Clustal Consensus

Poecilia formosa ELOVL8a Siganus canaliculatus ELOVL8a Danio rerio ELOVL8a Lepisosteus oculatus ELOVL8a Danio rerio ELOVL8b Astyanax mexicanus ELOVL8b Ictalurus punctatus ELOVL8b Poecilia formosa ELOVL8b Siganus canaliculatus ELOVL8b Clustal Consensus

Poecilia formosa ELOVL8a Siganus canaliculatus ELOVL8a Danio rerio ELOVL8a Lepisosteus oculatus ELOVL8a Danio rerio ELOVL8b Astyanax mexicanus ELOVL8b Ictalurus punctatus ELOVL8b Poecilia formosa ELOVL8b Siganus canaliculatus ELOVL8b clustal Consensus
MTGSNVVTKWQKLQLFYQGILENGDKRTDNWLLVYSPVPITCIFLCYLITIWAAGPKLMANRRPVNI RPVLVVYNFAMVCL ---MKALTMWQKLQLFYRGI LENGDKRTDDWLLVYSPVPISS I FLCYLI I IWLGPKLMAKRQPVNIKP ILIVYNFAMVCL -MVSVSPSTWQKLQILYERI LENGDKRTD GWLLVYSPLPVGGI FLCYLVMVWFGPKLMVHREPVNIQALLI IYNFSMVCL -MVSVSPSTWQKLQI LYERI LENGDKRTDGWLLVYSPLPVGGI FLCYLVMVW FGPKLMVHREPVN IQALLI IYNFSMVCL -----MESAWQRLE SMHKW IVENGDKRTDPWLLVYSPVPI ICI FLCYLGVIW I GPKLMKNMEPVNI KGLLIVYNFSMVGL ----MASAWQREESMHQW I LENGDKRTDPWLLVYS PVPVACI FLCYLGVLW IGPKLMKNREPLNIRVVLIVYNFAMVCL ----MESTWQRVESLHQW ILENGDKRTDPWLLVYS PVPVVLI FLFYLGILWLGPRLMRNRDPVDI KLVLIVYNFAMVCL ----MASAGNHVLSVHQWILENGDNRTDPWPLVYSPLPVTFI FLGYLCMIWVGSHLMKTRKPFEI KTVLIVYNFSMVGL -----MASAWEDVLSVYQSYLDNGDKRTDPWLLVYSPVPVALI FLVYLFVVWLGPRLMHHKQPVDI KAVLIVYNFAMVGL

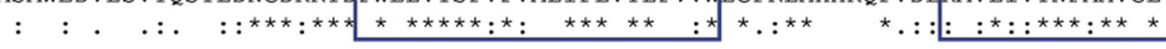

SAYMFYEFTASSWLAGYSLLCQPVDYSNNPLALRMARVCWWFYFSKVICVCVLQI FFILRKKN SQLTFLHVYHHATMIFN SAYMFYEFTASSWLARYSVLCQPVDYSSSPLAMRMARVCWWFYFSKVIELS-DT I FFILRKKNNQLTFLHVYHHATMI FN SAYMFYEFTASSWLASYSLLCQPVDYTENPLPMRMARVCWWFYFSKVIELA-DTMFFILRKKNNQLTFLHVYHHGTMI FN SAYMFYEFTASSWLASYSLLCQPVDYTENPLPMRMARVCWWFYFSKVIELA-DTMFFILRKKNNQLTFLHVYHHGTMI FN SVYMFHEFLVTSWLANYSYLCQPVDYSTSPLGMRMANVCWWFFFSKVIELS-DTVFFILRKKNSQLTFLHVYHHGTMIFN SVYMFHEFLVTSWLSNYSYLCQPVDYSTSPLAMRMASVCWWFFFSKVIELV-DTVFFILRKKNSQLTFLHIYHHGTMIFN SVYMFHEFLMTSWLSNYSYLCQPVDYSTGPLALRMARVCWWFFFSKVIELS-DTVFFILRKKNSOLTFLHVYHHGTMI FN SAYMFYEFLVTSWLSNYSLLCQPVDYSETPLPLRMASVCWWFFFSKIIELL-DTFFFVLRKKNSQLTFLHVYHHGTMIFN SAYMCYEFLVTSWLSNYSFLCQPVDYSSSPLAMRMARACWWFFFSKIIELS-DTIFFILRKKNSQVTFLHVYHHATMIFN

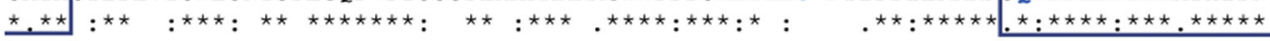

III

WWTGVKYVAGGO SFLIGLINSLVHIVMYLYYGLAALGPHMNKYLWWKQYLTSLQLLQFFVVTMHTAYNLHADCDFPDSMN WWA GVKYVAGGOSFLIGLINSLVHVVMYLYYGLAA FGPSMTKYLWWKRYLTCLQLLQFFIVTIHTIYNLFADCDFPDSMN WWAGVKYVAGGOSFLIGL INSFVHVVMYMYYGLAA GPQMQKYLWWKRYLTSLQLLQF FIVTIHTAFNLYADCDFPDSMN WWA GVKYVAGGOSFL I GL INSEVHVVMYMYYGLAA GPQMQKYLWWKRYLTSLQLLQFFIVTIHTAFNLYADCDF PDSMN WWAGVKYVAGGOSFFIGLLNTFVHIWMY SYYGLAA GPHLQKYLWWKRYLTSLQLVQFILLTVHTGYNLFTECEFPDSMN WWA GVKYVAGGOSFFIGLLNTFVHIVMYSYYGLAA GPHMQKYLWWKRYLTSLQLLQFVLLTTHTGYNLFTECDFPDSMN WWA GVKFVAGGOSFFIGLLNTEVHIIMY SYYGLAAFGPHMQRYLWWKRYLTSLQLLQFVLLTTHTGYNLFTECDFPDSMN WWA GVKYVAGGOSFFIGLVNTFVHIIMYTYYGLSAFGPHMQKHLWWKKYLTILQLLQELLFFLHTGYNLITECDFPDSMN WWA GIKYVAGGOSFFIGVVNSFVHVVMYSYYGLAA GPHMQKYLWWKRYITSLQLVQFVLFLVHTGYNLFAECDFPDSMN

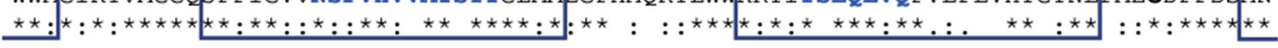
IV V

Fig. 2 Multiple alignments of Elovl8a and Elovl8b among fishes. Six conserved transmembrane $\alpha$-helix domains are boxed and labeled with I to VI, respectively. Four conserved motifs of elongases are marked in blue type. Cysteines are shown in bold type. ER retrieval signal at the C-terminal is signed by dotted box. Asterisks $(*)$ indicate conservation of the amino acids among these sequences. 
A

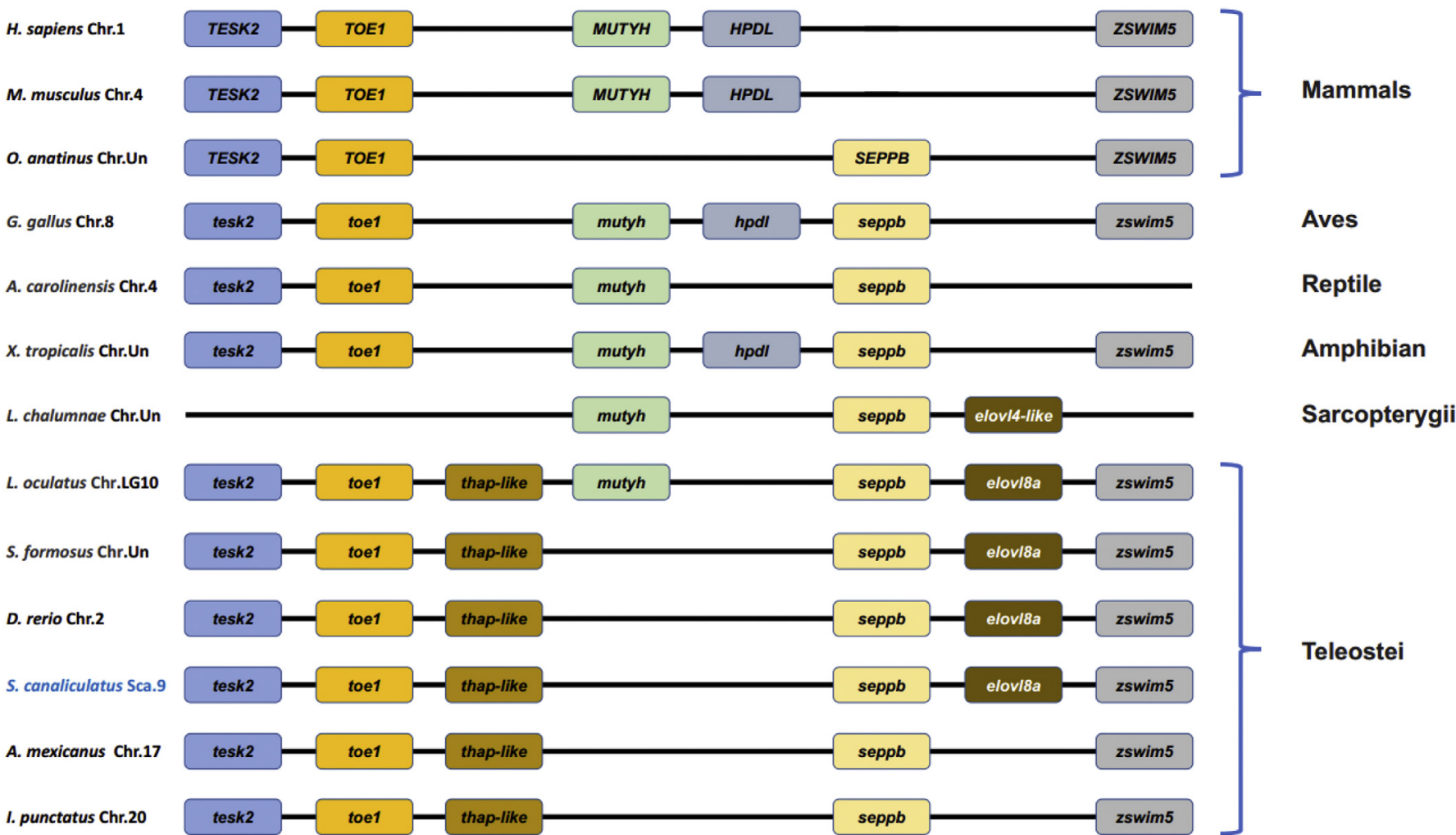

B

H. sapiens Chr.

M. musculus Chr.4
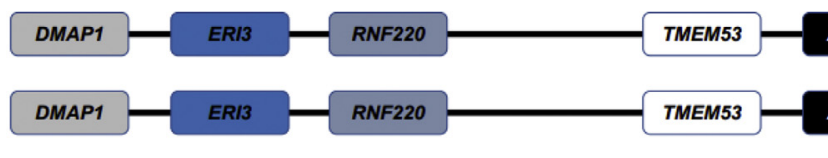

O. anatinus Chr.Un

G. gallus Chr.8

A. carolinensis Chr.4

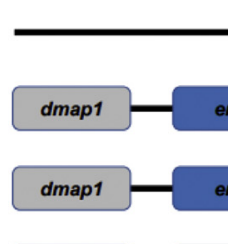

RNF220

F220

TMEM53
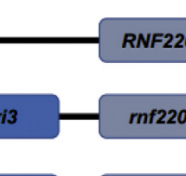

$r n f 220$

TMEM53

\section{ARMH1}

ARMH1

ARMH1

tmem53

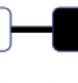

\section{ar}

eri3 rnf220 rnf220-like

tmem53

\section{armh1}

tmem53

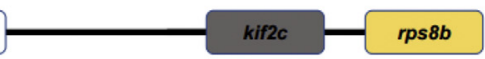

X. tropicalis Chr.Un

L. chalumnae Chr.Un

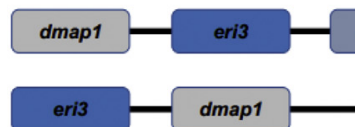

\section{rnf220}
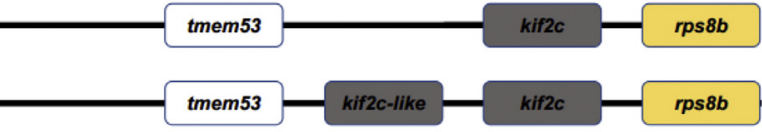

L. oculatus Chr.LG10

$$
d m
$$

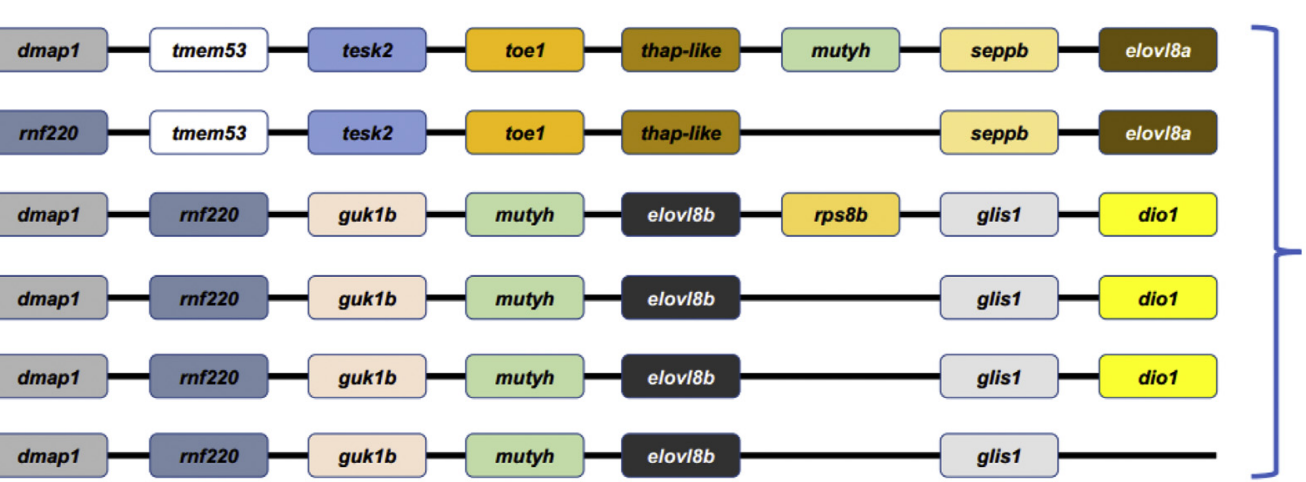

D. rerio Chr.8

S. canaliculatus Sca.19

I. punctatus Chr.5

A. mexicanus Chr.17

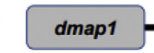

rnf220

guk1b mutyh
Mammals<smiles>C1CCCCC1</smiles>

Aves

Reptile

Amphibian

Sarcopterygii

Teleostei

Fig. 3. Synteny comparisons of the elovl8a (A) and elovl8b (B) genes in vertebrates. The colorful blocks represent different genes. The solid lines represent intergenic regions. The target species in present study are marked in blue type. 


\section{A}

L. oculatus elovl8a

\begin{tabular}{|l|l|l|l|l|l|l|l|l|l|l|l|l|l|l|l|l|l}
$58 \mathrm{bp}$ & $0.58 \mathrm{~kb}$ & $188 \mathrm{bp}$ & $1.57 \mathrm{~kb}$ & $81 \mathrm{bp}$ & $0.22 \mathrm{~kb}$ & $57 \mathrm{bp}$ & $0.10 \mathrm{~kb}$ & $115 \mathrm{bp}$ & $1.03 \mathrm{~kb}$ & $128 \mathrm{bp}$ & $0.48 \mathrm{~kb}$ & $158 \mathrm{bp}$ & $0.65 \mathrm{~kb}$ & $67 \mathrm{bp}$ \\
\hline
\end{tabular}

S. formosus elovl8a

\begin{tabular}{|l|l|l|l|l|l|l|l|l|l|l|l|l|l|l|l|l|l}
$58 \mathrm{bp}$ & $0.58 \mathrm{~kb}$ & $188 \mathrm{bp}$ & $0.24 \mathrm{~kb}$ & $81 \mathrm{bp}$ & $0.22 \mathrm{~kb}$ & $57 \mathrm{bp}$ & $0.09 \mathrm{~kb}$ & $115 \mathrm{bp}$ & $1.38 \mathrm{~kb}$ & $128 \mathrm{bp}$ & $1.03 \mathrm{~kb}$ & $168 \mathrm{bp}$ \\
\hline
\end{tabular}

D. rerio elovl8a

\begin{tabular}{|c|c|c|c|c|c|c|c|c|c|c|c|c|c|c|}
\hline $\begin{array}{l}198 \mathrm{bp} \\
(3 \mathrm{bp})\end{array}$ & $0.16 \mathrm{~kb}$ & 67 bp & $0.18 \mathrm{~kb}$ & 188 bp & $0.08 \mathrm{~kb}$ & 81 bp & $1.99 \mathrm{~kb}$ & 57 bp & $0.10 \mathrm{~kb}$ & $115 \mathrm{bp}$ & $2.56 \mathrm{~kb}$ & 128 bp & $0.08 \mathrm{~kb}$ & $\begin{array}{c}420 \mathrm{bp} \\
\text { (168 bp) }\end{array}$ \\
\hline $\begin{array}{c}276 \mathrm{bp} \\
\text { (6 bp) }\end{array}$ & $1.36 \mathrm{~kb}$ & $58 \mathrm{bp}$ & $0.15 \mathrm{~kb}$ & $188 \mathrm{bp}$ & $0.11 \mathrm{~kb}$ & $81 \mathrm{bp}$ & $0.11 \mathrm{~kb}$ & 57 bp & $0.20 \mathrm{~kb}$ & $115 \mathrm{bp}$ & $0.37 \mathrm{~kb}$ & $128 \mathrm{bp}$ & $1.17 \mathrm{~kb}$ & $\begin{array}{c}342 \mathrm{bp} \\
(171 \mathrm{bp})\end{array}$ \\
\hline
\end{tabular}

S. canaliculatus elovl8a

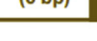

\section{B}

A. mexicanus elovl8b

\begin{tabular}{|c|c|c|c|c|c|c|c|c|c|c|c|c|c|c|}
\hline 456 bp & $1.90 \mathrm{~kb}$ & $\begin{array}{l}77 \mathrm{bp} \\
(58 \mathrm{bp} \text { ) }\end{array}$ & $.77 \mathrm{~kb}$ & $188 \mathrm{bp}$ & $0.64 \mathrm{~kb}$ & 81 bp & $0.16 \mathrm{~kb}$ & 57 bp & $1.77 \mathrm{~kb}$ & 115 bp & $0.13 \mathrm{~kb}$ & $128 \mathrm{bp}$ & $1.18 \mathrm{~kb}$ & $\begin{array}{l}1822 \mathrm{bp} \\
(168 \mathrm{bp})\end{array}$ \\
\hline
\end{tabular}

I. punctatus elovl8b

\begin{tabular}{|l|l|l|l|l|l|l|l|l|l|l|l|l|l|l|l|l|l}
\hline $422 \mathrm{bp}$ & $1.07 \mathrm{~kb}$ & $\begin{array}{c}85 \mathrm{bp} \\
(58 \mathrm{bp})\end{array}$ & $0.78 \mathrm{~kb}$ & $188 \mathrm{bp}$ & $2.19 \mathrm{~kb}$ & $81 \mathrm{bp}$ & $0.26 \mathrm{~kb}$ & $57 \mathrm{bp}$ & $0.34 \mathrm{~kb}$ & $115 \mathrm{bp}$ & $0.11 \mathrm{~kb}$ & $128 \mathrm{bp}$ & $0.10 \mathrm{~kb}$ & $\begin{array}{c}314 \mathrm{bp} \\
(168 \mathrm{bp})\end{array}$ \\
\hline
\end{tabular}

D. rerio elovl8b
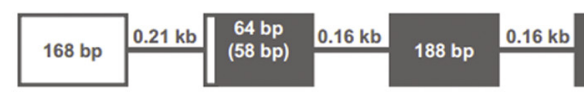

$81 \mathrm{kp} \quad 0.12 \mathrm{~kb}$

$57 \mathrm{bp} \quad 0.10 \mathrm{~kb}$

$115 \mathrm{bp} 0.80 \mathrm{~kb}$

$128 \mathrm{bp} \quad 0.18 \mathrm{~kb} \quad \begin{gathered}442 \mathrm{bp} \\ (168 \mathrm{bp})\end{gathered}$

S. canaliculatus elovl8b

\begin{tabular}{|c|c|c|c|c|c|c|c|c|c|c|c|c|c|c|}
\hline $326 \mathrm{bp}$ & $0.65 \mathrm{~kb}$ & $\begin{array}{c}71 \mathrm{bp} \\
\text { (58 bp) }\end{array}$ & $0.44 \mathrm{~kb}$ & $188 \mathrm{bp}$ & $0.12 \mathrm{~kb}$ & $81 \mathrm{bp}$ & $0.58 \mathrm{~kb}$ & 57 bp & $0.09 \mathrm{~kb}$ & 115 bp & $1.69 \mathrm{~kb}$ & $128 \mathrm{bp}$ & $0.08 \mathrm{~kb}$ & $\begin{array}{l}890 \mathrm{bp} \\
\text { (168 bp) }\end{array}$ \\
\hline
\end{tabular}

Fig. 4. Comparative analysis of the gene structures of elovl8a (A) and elovl8b (B) genes among different fish species. The colorful boxes and lines represent the exons and introns, respectively. Boxes in blank indicate the untranslated regions. Numbers in boxes and on lines represent the length of exons and introns, respectively. 


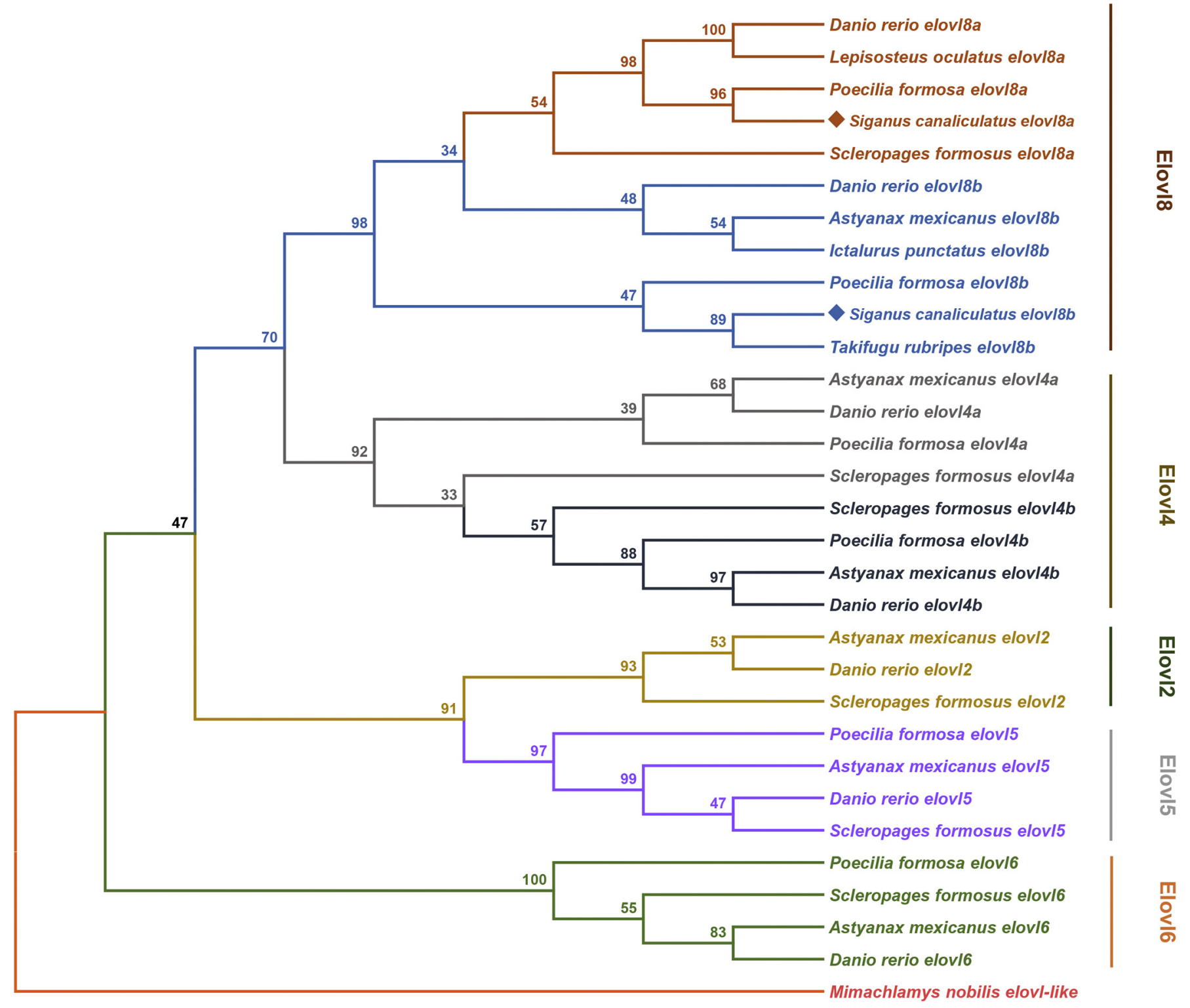

Fig. 5. Phylogenetic tree inferred the relationship of fish five elongase genes. The tree was constructed by Neighbor Joining (NJ) methods based on a dataset of amino acids. Values at the nodes represent bootstrap percentages from 1,000 replicates. The target species are marked by diamonds. Mimachlamys nobilis is regarded as the outgroup species

(A)

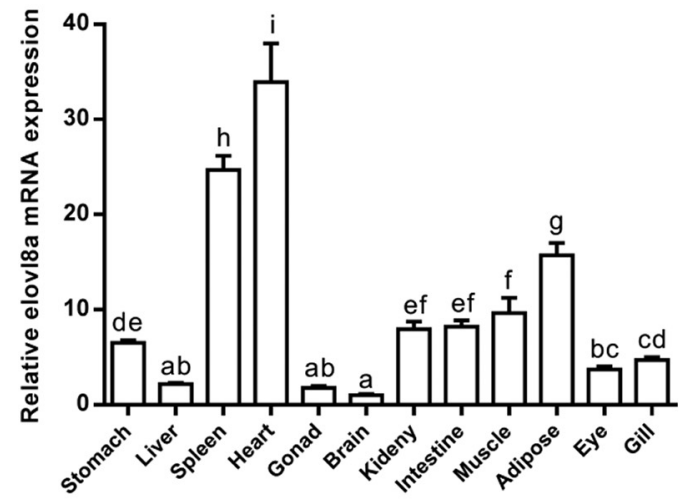

(B)

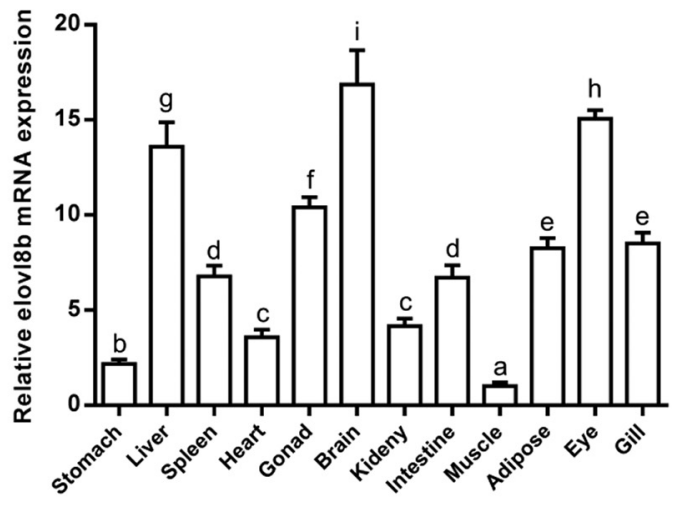

Fig. 6. Relative mRNA expression levels of elovl8a (A) and elovl8b (B) genes in different tissues of $S$. canaliculatus. Data are means \pm SEM ( $\mathrm{n}=6$ ). Different lowercase letters above the bars meant significant differences among different treatments $(\mathrm{P}<0.05)$. 
Fig. 7. Relative mRNA expression levels of elovl8a (A) and elovl8b (B) genes in SCHL incubated with different fatty acids for $24 \mathrm{~h}$. Data are means \pm SEM $(n=6)$. Different lowercase letters above the bars meant significant differences among different treatments $(\mathrm{P}<0.05)$

(A)

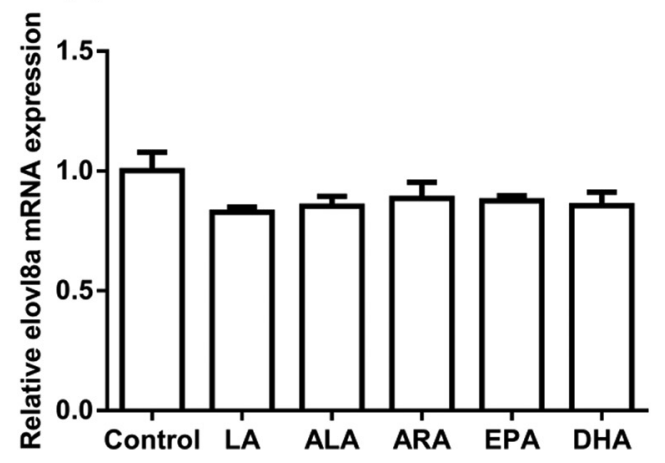

(B)

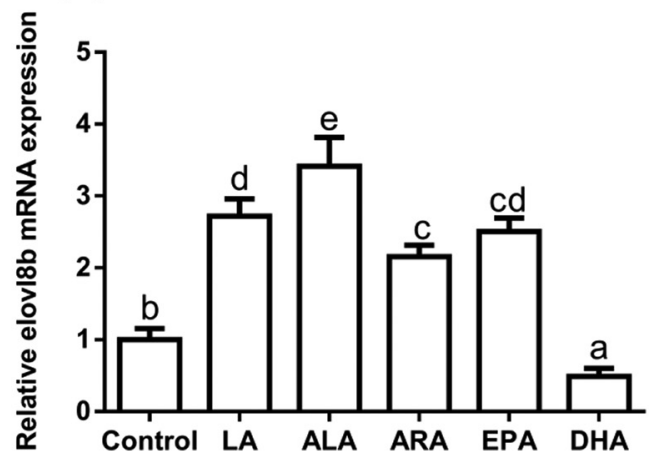


A

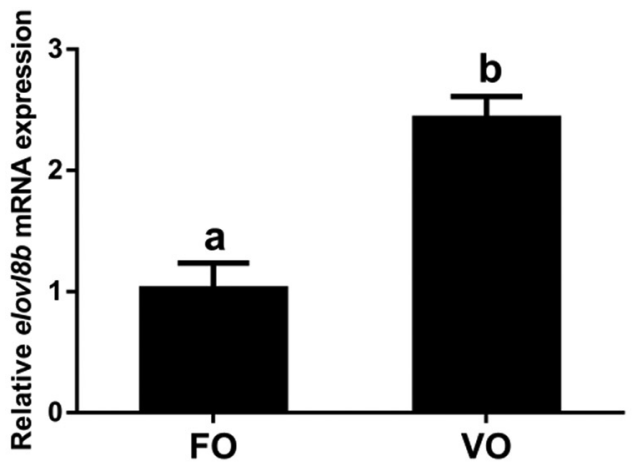

C

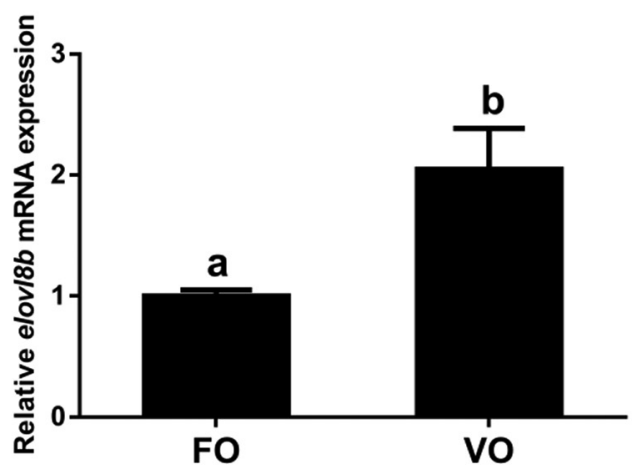

B

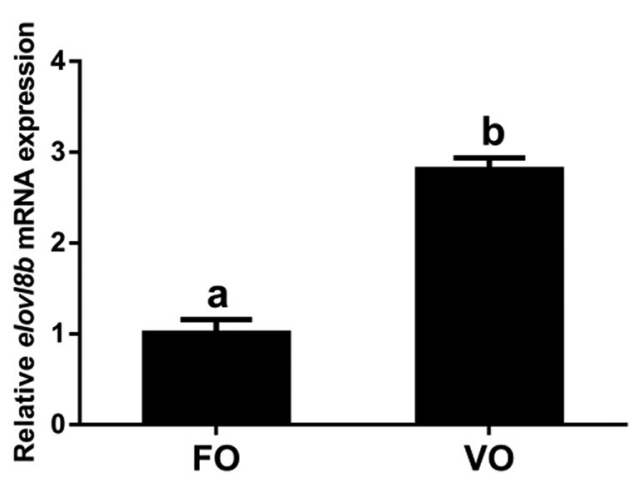

D

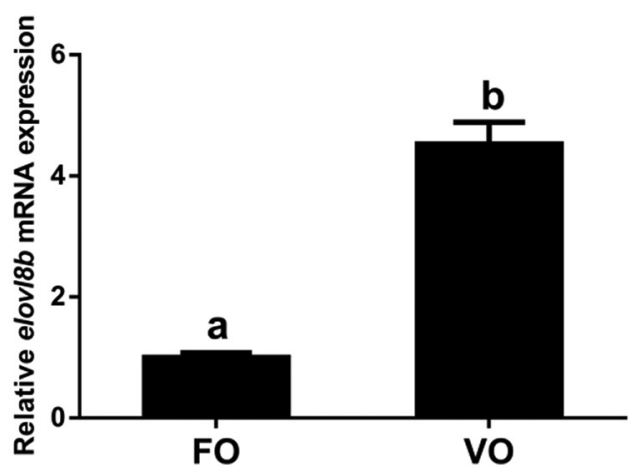

Fig. 8. The expressions of elovl $8 b$ mRNA in different tissues (A, brain; $\mathbf{B}$, liver; $\mathbf{C}$, intestine; $\mathbf{D}$, gill) of $S$. canaliculatus fed diets containing different lipid sources, respectively. Data are means \pm SEM $(n=6)$. Different lowercase letters above the bars meant significant differences among different treatments $(\mathrm{P}<0.05)$. 
Omega-6 Omega-3
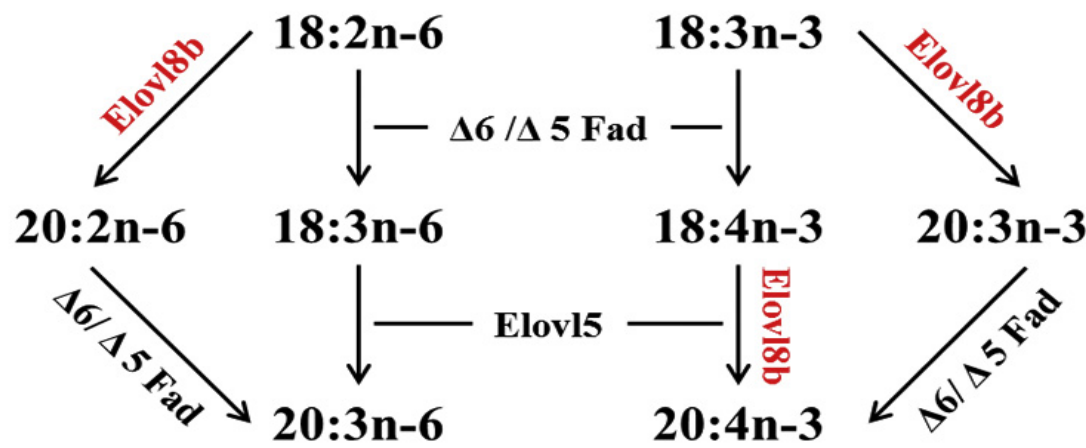

$\downarrow-\Delta 6 / \Delta 5$ Fad $-\downarrow$

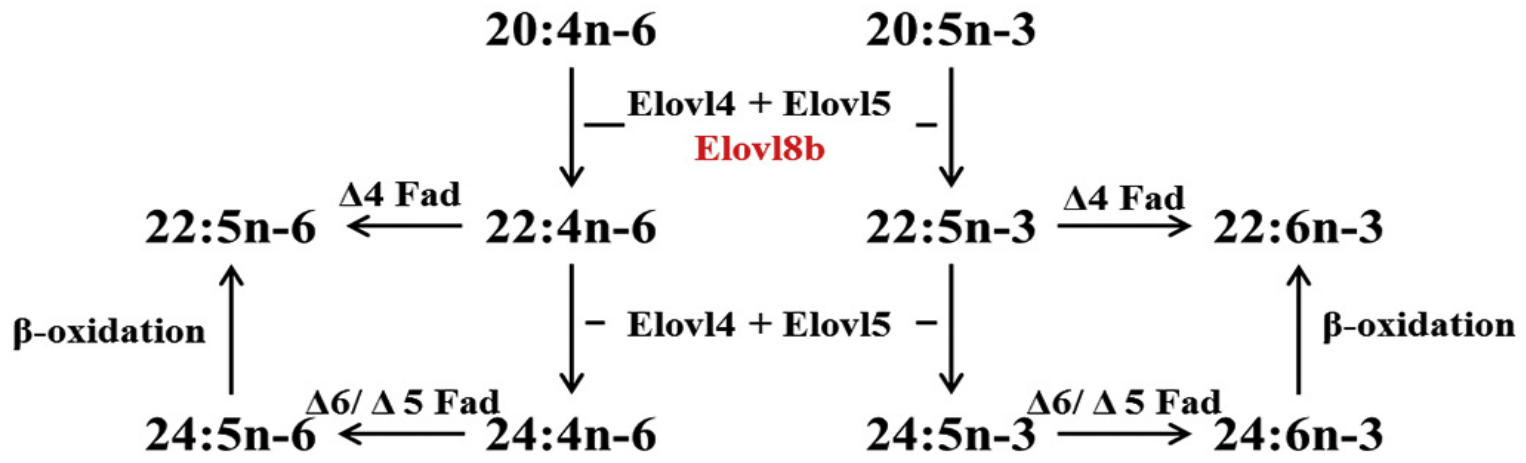

Fig. 9. The biosynthesis pathway of long-chain polyunsaturated fatty acids $(\leq C 24)$ from linoleic $(18: 2 n-6)$ and $\alpha$-linolenic (18:3n-3) acids in rabbitfish. Enzymatic activit ies shown in the scheme are predicted from heterologous expression in S. cerevisiae of the $\Delta 6 / \Delta 5$ fatty acyl desaturase $(\Delta 6 / \Delta 5 \mathrm{Fad}$ ), the $\Delta 4 \mathrm{Fad}$ ( $\mathrm{Li}$ et al., 2010), the Elovl4 and Elovl5 elongases (Monroig et al., 2012) and the herein reported Elovl8b elongases. 
Table 1. Functional characterizations of rabbit fish elovl8 elongases: conversions on polyunsaturated fatty acid (FA) substrates.

\begin{tabular}{lllll}
\hline FA substrate & Product & Elolv8a & Elolv8b & Activity \\
\hline $18: 2 \mathrm{n}-6$ & $20: 2 \mathrm{n}-6$ & 0 & 2.0 & $\mathrm{C} 18 \rightarrow 20$ \\
$18: 3 \mathrm{n}-3$ & $20: 3 \mathrm{n}-3$ & 0 & 3.7 & $\mathrm{C} 18 \rightarrow 20$ \\
$18: 4 \mathrm{n}-3$ & $20: 4 \mathrm{n}-3$ & 0 & 3.7 & $\mathrm{C} 18 \rightarrow 20$ \\
$20: 4 \mathrm{n}-6$ & $22: 4 \mathrm{n}-6$ & 0 & 2.0 & $\mathrm{C} 20 \rightarrow 22$ \\
$20: 5 \mathrm{n}-3$ & $22: 5 \mathrm{n}-3$ & 0 & 3.2 & $\mathrm{C} 20 \rightarrow 22$ \\
\hline
\end{tabular}

Results are expressed as a percentage of total FA substrate converted to elongated product. 


\section{Author Contributions}

Conceptualization, Yuanyou Li and Shuqi Wang; Methodology, Yang Li and Zhengyong Wen; Software, Yang Li and Cuihong You; Validation, Yueling Zhang and Zhiyong Xie; Formal analysis, Douglas R. Tocher and Yueling Zhang; Investigation, Yang Li and Zhengyong Wen; Resources, Cuihong You and Zhiyong Xie; Data curation, Yang Li and Zhengyong Wen; Writing-original draft, Yang Li and Zhengyong Wen; Writing-review and editing, Douglas R. Tocher, Yuanyou Li and Shuqi Wang; Visualization, Yang Li and Zhengyong Wen; Supervision, Yuanyou Li and Shuqi Wang; Project administration, Yuanyou Li and Shuqi Wang; Funding acquisition, Yuanyou Li and Shuqi Wang. 


\section{Highlights}

1. Two novel fish-specific elovl8 genes were first identified in Siganus canaliculatus.

2. Comprehensive studies revealed two elovl8 isoforms were widely existed in teleost.

3. Rabbitfish elovl8a was highly expressed in heart and spleen, while elovl $8 b$ was mainly distributed in the brain and eye.

4. Elov18b but not Elovl8a was found to have the ability to elongate the LC-PUFA.

5. A new mechanism involved in LC-PUFA biosynthesis was established. 